J. DIFFERENTIAL GEOMETRY

45 (1997) 288-318

\title{
PRIMITIVE CALABI-YAU THREEFOLDS
}

\author{
MARK GROSS
}

\section{Introduction}

A Calabi-Yau threefold is a complex projective threefold $X$ (possibly with some suitable class of singularities, say terminal or canonical) with $\omega_{X} \cong \mathcal{O}_{X}$ and $h^{1}\left(\mathcal{O}_{X}\right)=h^{2}\left(\mathcal{O}_{X}\right)=0$. One of the fundamental gaps in the classification of algebraic threefolds is the lack of understanding of Calabi-Yau threefolds. Here I will try to set forth a program to bring the morass of thousands of examples of Calabi-Yaus under control.

The ideas here go back to the papers of Friedman [4] and Reid [27]. Friedman studied smoothability of Calabi-Yau threefolds with ordinary double points. Based on these results, Reid conjectured that there could perhaps be a single irreducible moduli space of (non-Kähler) Calabi-Yau threefolds, such that any Calabi-Yau threefold is the small resolution of a degeneration of this family to something with ordinary double points. So one can think of all the chaos of the algebraic examples as simply being "boundary phenomena" for the moduli space of this master Calabi-Yau. I suspect the most difficult part of this conjecture, often known as Reid's fantasy, will be passing from algebraic to non-algebraic threefolds. We do not understand how to deal with non-Kähler Calabi-Yau threefolds or find non-algebraic contractions.

Unlike in the K3 case, where it is possible to deform an algebraic K3 surface to a non-algebraic one, the deformation of a projective CalabiYau threefold, even singular, is still projective. So it makes sense to insist on staying within the projective category. Reid's picture given

Received December 8, 1995. Author was supported in part by NSF grant DMS9400873 
above needs to be modified if we restrict attention to projective threefolds; indeed, an algebraic Calabi-Yau of Picard number 1 has no algebraic birational contractions, and so cannot be the resolution of another algebraic Calabi-Yau threefold. Since there are many examples of Calabi-Yau threefolds with Picard number 1, we cannot hope that all Calabi-Yau threefolds arise as resolutions of degenerations of a single family of algebraic threefolds. Nevertheless, a somewhat weaker picture seems reasonable.

As proposed in [2], [5] and [6], we can think of the moduli of CalabiYaus as forming a giant web, a directed graph where each node is a deformation class of Calabi-Yau threefolds. We draw an arrow $\mathcal{M}_{1} \rightarrow$ $\mathcal{M}_{2}$ if for the general element $\tilde{X}$ of deformation class $\mathcal{M}_{1}$, there is a birational contraction morphism $\pi: \tilde{X} \rightarrow X$ and a flat family $\mathcal{X} \rightarrow$ $(\Delta, 0)$ such that $\mathcal{X}_{0} \cong X$ and $\mathcal{X}_{t} \in \mathcal{M}_{2}$ for general $t \in \Delta$. For example, let $\mathcal{M}_{Q}$ be the moduli space of smooth quintics in $\mathbf{P}^{4}$, and $\mathcal{M}_{D}$ the moduli space of double covers of $\mathbf{P}^{3}$ branched over smooth octics. Let $T$ be the blowup of a quintic with a triple point; let $\mathcal{M}_{T}$ be the moduli space of such $T$. We have two contraction morphisms $\pi_{1}: T \rightarrow T_{1}$, $\pi_{2}: T \rightarrow T_{2}$ with $\pi_{1}$ the contraction of the exceptional cubic surface to $T_{1}$, a quintic with a triple point, and $\pi_{2}$ the Stein factorization of the projection $T \rightarrow \mathbf{P}^{3}$ from the triple point. $T_{1}$ can be smoothed to a smooth quintic, and $T_{2}$ can be smoothed to a double cover of $\mathbf{P}^{3}$ branched over a smooth octic. Thus a tiny portion of our web will be:

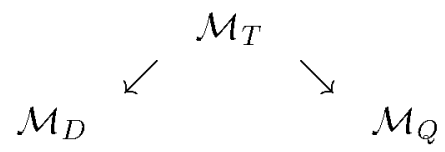

By taking deformation classes of all simply-connected Calabi-Yau threefolds, we get an enormous (perhaps infinite) graph. So one question that immediately comes up is often thought of as one version of Reid's fantasy:

The Connectedness Conjecture 0.1. The graph of simply connected Calabi-Yaus is connected.

The evidence for this is strictly experimental at this point: large classes of examples have been connected up, "by hand," e.g. [6], where moduli of Calabi-Yau complete intersections in products of projective spaces are connected up. Joint work with T.-M. Chiang, B. Greene and Y. Kanter has connected up all known examples of Calabi-Yaus in weighted $\mathbf{P}^{4}([3])$ and similar results were obtained independently in [1]. 
Clearly, the nodes at the bottom of this Calabi-Yau graph, such as $\mathcal{M}_{Q}$ and $M_{D}$, will be the Calabi-Yaus which play the role of Reid's master Calabi-Yau in the algebraic setting. I call a Calabi-Yau whose deformation class is a node of the graph with no outgoing arrows a primitive Calabi-Yau. More formally,

Definition 0.2. A non-singular Calabi-Yau threefold $\tilde{X}$ is primitive if there is no birational contraction $\tilde{X} \rightarrow X$ with $X$ smoothable to a Calabi-Yau threefold which is not deformation equivalent to $\tilde{X}$.

So one approach to proving the connectedness conjecture would be to classify primitive Calabi-Yaus and then to find some paths through the Calabi-Yau graph connecting the primitive Calabi-Yaus together.

Beyond the connectedness conjecture, we might learn a lot more about the number of families of Calabi-Yaus as a whole if we can understand the class of primitive Calabi-Yaus. Four further questions of varying strength are

\section{Questions 0.3.}

(1) Are there a finite number of flat families $\mathcal{X}_{i} \rightarrow \mathcal{S}_{i}$ of non-singular Calabi-Yau threefolds such that for any Calabi-Yau $\tilde{X}$, there is a flat family $\mathcal{X} \rightarrow \Delta$ with $\mathcal{X}_{t}$ in one of the families $\mathcal{X}_{i} \rightarrow \mathcal{S}_{i}$ for $t \neq 0$ and $\mathcal{X}_{0}$ birational to $\tilde{X}$ ?

(2) The same question, except we insist that $\tilde{X}$ be a crepant resolution of $\mathcal{X}_{0}$.

(3) Are there a finite number of flat families $\mathcal{X}_{i} \rightarrow \mathcal{S}_{i}$ of Calabi-Yau threefolds with canonical singularities such that any Calabi-Yau $\tilde{X}$ is birational to some member $X$ of one of these families?

(4) Same question as (3), except we insist that $\tilde{X}$ be a crepant resolution of $X$.

Intuitively, these should follow if there are only a finite number of families of primitive Calabi-Yau threefolds. Unfortunately, none of them do immediately, though (1) follows if fourfold flops and Qfactorializations exist. We will discuss these questions in $\S 3$, and in particular the current technical obstacles involved in answering these questions, even under the assumption that there are only a finite number of families of primitive Calabi-Yaus. Note that (3) implies there are only a finite number of families up to birational equivalence, which has 
been proven in the case of elliptic Calabi-Yau threefolds in [8]. (4) implies the stronger result that there are only a finite number of families up to biregular equivalence, since a Calabi-Yau with canonical singularities has only a finite number of crepant resolutions.

So the main philosophy espoused by this paper is that we should attempt to classify primitive Calabi-Yaus, and that this should be easier than classifying all Calabi-Yaus. Is there any hope for such a classification? Well, obviously any Picard number 1 Calabi-Yau is primitive, and we do not have a classification of Picard number 1 Calabi-Yaus, but at least I only know of about 30 such threefolds, as opposed to an order of 10000 currently known examples of Calabi-Yaus in general. Suppose we could understand Picard number one Calabi-Yaus. The hope then would be that there are very few primitive Calabi-Yaus with Picard number greater than one. There certainly are some: the bidegree $(3,3)$ hypersurface in $\mathbf{P}^{2} \times \mathbf{P}^{2}$ furnishes an example, since it has no algebraic contractions, and there are other, less trivial examples. However, we shall give some evidence in $\S 2$ that we should not expect many such examples.

How do we attack this classification problem? Given a Calabi-Yau $\tilde{X}$, we presumably need to understand birational contractions of $\tilde{X}$, $\pi: \tilde{X} \rightarrow X$, and smoothability of $X$. Now $X$ will have canonical singularities, and these can be very complicated. So we cannot really hope to completely answer the question of when $X$ is smoothable. However, we can answer this question if we assume $\pi$ is a primitive contraction, i.e., $\pi$ cannot be factored in the algebraic category. We have already begun this study in [10], where types I and II contractions were studied (small contractions and divisorial contractions to points respectively). However, type III contractions still need to be considered. These are contractions which contract a divisor to a curve. Technically, this is a harder case to deal with, and we devote $\S 1$ to it. The final result, however, is remarkably similar to Theorem 5.8 of [10]. We have

Theorem 0.4. Let $\pi: \tilde{X} \rightarrow X$ be a primitive type III contraction, contracting a divisor $E$ to a curve $C$. Then $X$ is smoothable unless $C \cong \mathbf{P}^{1}$ and $E^{3}=7$ or 8 .

This follows from Theorems 1.3 and 1.7.

Recall that on a Calabi-Yau threefold, $E^{3}$ is the self-intersection of the canonical class of $E$. Thus, if $E$ is a normal surface, $C \cong \mathbf{P}^{1}$, then $E^{3}=8$ is the case that $E$ is a minimal scroll and $E^{3}=7$ is the case that $E$ is a minimal scroll blown up in one point. 
Combining this with the results of [10], we see that if $\tilde{X}$ is a primitive Calabi-Yau and $\pi: \tilde{X} \rightarrow X$ is a primitive contraction, then $\pi$ is either a contraction of a single $\mathbf{P}^{1}$ with normal bundle $\mathcal{O}_{\mathbf{P}^{1}}(-1) \oplus \mathcal{O}_{\mathbf{P}^{1}}(-1)$, or a contraction of $\mathbf{P}^{2}$, a minimal ruled surface over $\mathbf{P}^{1}$, such a surface blown up in one point, or a non-normal surface with $E^{3}=7$. This in fact turns out to be a very strong restriction, principally since then the exceptional divisor $E$ of any primitive divisorial contraction on a primitive Calabi-Yau satisfies $c_{2} . E<0$. We shall take this up in $\S 2$. There we will derive some immediate combinatorial consequences of these results, and speculate what still needs to be done. I believe that a more detailed combinatorial analysis will yield far stronger results then are obtained in $\$ 2$. I hope to treat this approach in a future paper.

Finally, we note that connecting together moduli spaces of CalabiYau threefolds appears to be playing an important role in physics. In particular, in [7], a physical explanation has been given for transitions between moduli spaces. So far only degenerations involving ordinary double points have been studied, but there is apparently no physical reason for restricting attention to such cases.

I would like to thank P.M.H. Wilson for many helpful conversations during the preparation of this paper.

\section{Type III contractions}

Theorem 1.1. Let $\pi: \tilde{X} \rightarrow X$ be a primitive type III contraction of a non-singular Calabi-Yau threefold $\tilde{X}$, contracting an exceptional divisor $E$ to a curve $C$. Then the following hold:

(a) $C$ is a non-singular curve.

(b) $\pi: E \rightarrow C$ is a conic bundle over $C$, and each fibre is either a non-singular conic, a union of two lines meeting at a point, or a doubled line. If the general fibre is a non-singular curve, then $E$ is normal. In this case, the singularities which appear on $E$ are $A_{n}$ $(n \geq 0)$ singularities at the singular point of a reducible reduced fibre, or two $A_{1}$ singularities on a non-reduced fibre.

Proof. For (a), see [36]; for (b), see [32], Theorem 2.2, keeping in mind [33]. q.e.d.

Proposition 1.2. Let $\pi: \tilde{X} \rightarrow X$ be a primitive type III contraction of a non-singular Calabi-Yau threefold $\tilde{X}$, contracting a divisor $E$ to a 
curve $C$. Let $\tilde{E}$ be the normalization of $E, f: \tilde{E} \rightarrow \tilde{X}$ the induced map, and $\tilde{E} \rightarrow \tilde{C} \rightarrow C$ the Stein factorization. Then the image of the natural map Def $(f) \rightarrow \operatorname{De} f(\tilde{X})$ has codimension $\geq p_{a}(\tilde{C})$.

Proof. If $E$ is already normal, then this is the result of [19, Prop. 6.5.] If $E$ is not normal, then the fibres of $\pi: \tilde{E} \rightarrow C$ are line pairs or doubled lines, and $\tilde{\pi}: \tilde{E} \rightarrow \tilde{C}$ is a $\mathbf{P}^{1}$ fibration with a section. Thus $\tilde{E}$ is a non-singular scroll. We proceed as in [33].

Define $N_{f}$ by the exact sequence

$$
0 \rightarrow \mathcal{T}_{\tilde{E}} \rightarrow f^{\star} \mathcal{T}_{\tilde{X}} \rightarrow N_{f} \rightarrow 0
$$

$N_{f}$ is torsion free, and fails to be locally free precisely at the inverse images of the pinch points of $E$. Thus $N_{f}^{\vee v}$ is locally free and $c_{1}\left(N_{f}\right)=$ $c_{1}\left(N_{f}^{\vee v}\right)$, showing that $N_{f}^{\vee v} \cong \omega_{\tilde{E}}$.

If $T_{f}^{1}$ is the tangent space to $\operatorname{Def}(f)$, we have an exact sequence by [25]:

$$
\begin{aligned}
H^{0}\left(\mathcal{T}_{\tilde{X}}\right) \oplus H^{0}\left(\mathcal{T}_{\tilde{E}}\right) & \rightarrow H^{0}\left(f^{*} \mathcal{T}_{\tilde{X}}\right) \rightarrow T_{f}^{1} \\
& \rightarrow H^{1}\left(\mathcal{T}_{\tilde{X}}\right) \oplus H^{1}\left(\mathcal{T}_{\tilde{E}}\right) \rightarrow H^{1}\left(f^{*} \mathcal{T}_{\tilde{X}}\right)
\end{aligned}
$$

This induces an exact sequence

$$
T_{f}^{1} \stackrel{\alpha}{\longrightarrow} H^{1}\left(\mathcal{T}_{\tilde{X}}\right) \stackrel{\beta}{\longrightarrow} H^{1}\left(N_{f}\right)
$$

where $\alpha$ is the differential of the map $\operatorname{De} f(f) \rightarrow \operatorname{De} f(\tilde{X})$ and $\beta$ is induced by $H^{1}\left(\mathcal{T}_{\tilde{X}}\right) \rightarrow H^{1}\left(f_{*} f^{*} \mathcal{T}_{\tilde{X}}\right)=H^{1}\left(f^{*} \mathcal{T}_{\tilde{X}}\right) \rightarrow H^{1}\left(N_{f}\right)$. Since $H^{0}\left(N_{f}\right) \subseteq H^{0}\left(\omega_{\tilde{E}}\right)=0, \alpha$ is injective. Thus, if $\operatorname{dim} \operatorname{im} \beta \geq p_{a}(\tilde{C})$, then $\operatorname{codim}(\operatorname{im}(\operatorname{Def}(f) \rightarrow \operatorname{Def}(\tilde{X}))) \geq p_{a}(\tilde{C})$. The composed map

$$
H^{1}\left(\mathcal{T}_{\tilde{X}}\right) \rightarrow H^{1}\left(N_{f}\right) \rightarrow H^{1}\left(N_{f}^{\vee \vee}\right)=H^{1}\left(\omega_{\tilde{E}}\right)
$$

is the natural map $H^{1}\left(\Omega_{\tilde{X}}^{2}\right) \rightarrow H^{1}\left(\Omega_{\tilde{E}}^{2}\right)$. Since $h^{1}\left(\omega_{\tilde{E}}\right)=p_{a}(\tilde{C})$, it is sufficient to show that $H^{1}\left(\Omega_{\tilde{X}}^{2}\right) \rightarrow H^{1}\left(\Omega_{\tilde{E}}^{2}\right)$ is surjective, or equivalently by Hodge theory, that $H^{2}\left(\Omega_{\tilde{X}}^{1}\right) \rightarrow H^{2}\left(\Omega_{\tilde{E}}^{1}\right)$ is surjective.

To show surjectivity of this map, note that we have exact sequences

$$
0 \rightarrow \mathcal{F}_{1} \rightarrow \Omega_{\tilde{X}}^{1} \rightarrow f_{*} f^{*} \Omega_{\tilde{X}}^{1} \rightarrow \mathcal{F}_{2} \rightarrow 0
$$

where $\mathcal{F}_{2}$ has support on the singular curve of $E$, and

$$
0 \rightarrow \mathcal{F}_{3} \rightarrow f^{*} \Omega_{\tilde{X}}^{1} \rightarrow \Omega_{\tilde{E}}^{1} \rightarrow \mathcal{F}_{4} \rightarrow 0
$$


where $\mathcal{F}_{4}$ has support on the pinch points of $\tilde{E}$. (1.2.2) shows that $H^{2}\left(f^{*} \Omega_{\tilde{X}}^{1}\right) \rightarrow H^{2}\left(\Omega_{\tilde{E}}^{1}\right)$ is surjective, and (1.2.1) shows that $H^{2}\left(\Omega_{\tilde{X}}^{1}\right) \rightarrow$ $H^{2}\left(f^{*} \Omega_{\tilde{X}}^{1}\right)$ is surjective if $H^{3}\left(\mathcal{F}_{1}\right)=0$. Now $H^{3}\left(\mathcal{F}_{1}\right) \cong H^{0}\left(\mathcal{F}_{1} \vee\right)^{\vee}$ by Serre duality, and there is an injection $\pi_{*} \mathcal{F}_{1} \vee \rightarrow \mathcal{T}_{X}$ since $\pi_{*} \mathcal{F}_{1} \vee$ is torsion-free, $\pi_{*} \mathcal{F}_{1}{ }^{\vee}$ and $\mathcal{T}_{X}$ coincide off a codimension 2 set, and $\mathcal{T}_{X}$ is reflexive. Thus $H^{0}\left(\mathcal{F}_{1} \vee\right) \subseteq H^{0}\left(\mathcal{T}_{X}\right)=0$ by $[13,8.6]$. We conclude that $H^{2}\left(\Omega_{\tilde{X}}^{1}\right) \rightarrow H^{2}\left(\Omega_{\tilde{E}}^{1}\right)$ is surjective, and the proposition follows. q.e.d.

Theorem 1.3. Let $\pi: \tilde{X} \rightarrow X$ be a primitive type III contraction of a non-singular Calabi-Yau threefold $\tilde{X}$, contracting a divisor $E$ to a curve $C$. If $p_{a}(C) \geq 1$, then $X$ is smoothable.

Proof. Let $f: \tilde{\mathcal{X}} \rightarrow \Delta$ be a deformation of $\tilde{X}$ over a contractible base $\Delta$ which is sufficiently general, so that the exceptional divisor $E$ does not deform to general $\tilde{\mathcal{X}}_{t}, t \in \Delta$. By Proposition 1.2, such exists. The contraction $\pi: \tilde{X} \rightarrow X$ yields a contraction $\tilde{\mathcal{X}} \rightarrow \mathcal{X}$ over $\Delta$ extending $\pi$. The general contraction $\tilde{\mathcal{X}}_{t} \rightarrow \mathcal{X}_{t}$ is then a small primitive contraction, and by [10, Proposition 5.1], $\mathcal{X}_{t}$ is smoothable unless $\mathcal{X}_{t}$ has exactly one ordinary double point. Thus it is enough to show that for general $t$, the singular locus of $\mathcal{X}_{t}$ is not exactly one ordinary double point. Note we are not excluding the possibility that $\tilde{\mathcal{X}}_{t}=\mathcal{X}_{t}$ for $t$ general, but in this case we are done. (This can happen if $p_{a}(C)=1$; see [32].)

First suppose that $E$ is normal and $\pi: E \rightarrow C$ has a singular fibre. By Theorem $1.1 \mathrm{~b}$ ), this singular fibre is either two $\mathbf{P}^{1}$ 's or a doubled $\mathbf{P}^{1}$. Let $Z$ be the homology class of either one of these $\mathbf{P}^{1}$ s in the first case or of the reduced fibre in the second case. If, for general $t \in \Delta$, $\mathcal{X}_{t}$ had only one ODP, then $\tilde{\mathcal{X}}_{t}$ would contain precisely one $\mathbf{P}^{1}$ with normal bundle $\mathcal{O}_{\mathbf{P}^{1}}(-1) \oplus \mathcal{O}_{\mathbf{P}^{1}}(-1)$ in the homology class of $Z$. This then implies that if we deform the complex structure of $\tilde{X}$ to a generic almost complex structure, the homology class $Z$ represents only one pseudo-holomorphic curve. But this contradicts [36, Lemma 4.1]. Thus for general $t, \mathcal{X}_{t}$ has worse singularities than one ODP.

Now suppose $E$ is either normal and $\pi: E \rightarrow C$ has no singular fibres, or $E$ is non-normal. If, in the notation of Proposition 1.2, $p_{a}(\tilde{C})=$ 1 , then by [32, Proposition 4.4] and [33], $\tilde{\mathcal{X}}_{t} \rightarrow \mathcal{X}_{t}$ is an isomorphism for general $t \in \Delta$; thus $X$ is smoothable. If $p_{a}(\tilde{C}) \geq 2$, let $l$ be the class of a fibre of $\tilde{E} \rightarrow \tilde{C}$, and let $\tilde{\mathcal{X}} \rightarrow \operatorname{Def}(\tilde{X})$ be the Kuranishi family of $\tilde{X}$. Let $S \rightarrow \operatorname{Def}(\tilde{X})$ be the irreducible component of the relative Douady space of $\tilde{\mathcal{X}} \rightarrow \operatorname{Def}(\tilde{X})$ corresponding to deformations of $l$. Now $\operatorname{dim} S \geq \chi\left(N_{l / \tilde{X}}\right)=\operatorname{dim} \operatorname{Def}(\tilde{X})$, but on the other hand, 
by Proposition 1.2 , the locus in $\operatorname{Def}(\tilde{X})$ where $S \rightarrow \operatorname{Def}(\tilde{X})$ has onedimensional fibres is codimension at least 2. Thus $S \rightarrow \operatorname{De} f(\tilde{X})$ must be surjective. Suppose that this map is generically one-to-one. Then $S_{\text {red }} \rightarrow \operatorname{Def}(\tilde{X})$ is a birational map, and so the one-dimensional fibres must be rational. This contradicts $p_{a}(\tilde{C}) \geq 2$. Hence again, for general $t \in \operatorname{Def}(\tilde{X}), \tilde{\mathcal{X}}_{t} \rightarrow \mathcal{X}_{t}$ is a small contraction contracting at least two curves. q.e.d.

We now focus on the necessary local deformation-theoretic calculations for the case that $C \cong \mathbf{P}^{1}$; in this case, the exceptional divisor $E$ always deforms with $\tilde{X}$.

Theorem 1.4. Let $\pi: \tilde{X} \rightarrow X$ be a primitive type III contraction of a non-singular Calabi-Yau threefold $\tilde{X}$, contracting a divisor $E$ to a curve $C \cong \mathbf{P}^{1}$. Suppose furthermore that $\tilde{X}$ is general in its moduli. Then the following hold:

(a) If $E^{3} \neq 7$, then $E$ is a normal surface.

(b) $\mathcal{I}_{C} / \mathcal{I}_{C}^{2}$ is a locally free sheaf on $C$ of rank 3 and degree $E^{3}-2$.

(c) Let $\mathbf{T}^{1}=\underline{\operatorname{Ext}}_{\mathcal{O}_{X}}^{1}\left(\Omega_{X}^{1}, \mathcal{O}_{X}\right)$. If $E$ is normal, then there are exact sequences

$$
0 \rightarrow R^{1} \pi_{* \underline{\operatorname{Hom}}_{\mathcal{O}}}\left(\pi^{*} \Omega_{X}^{1}, \mathcal{O}_{\tilde{X}}\right) \rightarrow \mathbf{T}^{1} \rightarrow \pi * \underline{\operatorname{Ext}}_{\mathcal{O}_{\tilde{X}}}^{1}\left(\pi^{*} \Omega_{X}^{1}, \mathcal{O}_{\tilde{X}}\right) \rightarrow 0
$$

and

$$
0 \rightarrow \mathcal{F} \rightarrow \pi_{*} \underline{\operatorname{Ext}}_{\mathcal{O}_{\tilde{X}}}^{1}\left(\pi^{*} \Omega_{X}^{1}, \mathcal{O}_{\tilde{X}}\right) \rightarrow \mathcal{O}_{C}\left(4-E^{3}\right) \rightarrow 0,
$$

where both $R^{1} \pi_{*} \underline{\operatorname{Hom}}_{\mathcal{O}_{\tilde{X}}}\left(\pi^{*} \Omega_{X}^{1}, \mathcal{O}_{\tilde{X}}\right)$ and $\mathcal{F}$ are sheaves of finite length, with

$$
\text { length }(\mathcal{F}) \geq 8-E^{3} \text {. }
$$

Proof. (a) If $E$ is not normal, then following the notation of Proposition 1.2 , we can assume that $p_{a}(\tilde{C})=0$ since $\tilde{X}$ is general in its moduli. Thus $\tilde{C} \rightarrow C$ is a double cover branched in precisely two points. By [36, Prop. 3.2], the map $\tilde{E} \rightarrow X$ has precisely two pinch points. As pointed out at the end of the proof of [36, Prop. 4.2], this implies that $E^{3}=7$. The same calculation as in [10, Theorem 5.2] also shows that $E^{3}=7$. 
(b) Let $\mathcal{I}_{C}$ be the ideal sheaf of $C \subseteq X$. We first need to understand $\mathcal{I}_{C} / \mathcal{I}_{C}^{2}$. In a neighborhood of a point of $C$, we can embed $X \subseteq Y$, with $Y$ smooth of dimension 4, since $X$ has only hypersurface singularities. Since $X$ is singular along $C, \mathcal{I}_{X / Y} \subseteq \mathcal{I}_{C / Y}^{2}$, and so $\mathcal{I}_{C} / \mathcal{I}_{C}^{2}=\mathcal{I}_{C / Y} /\left(\mathcal{I}_{C / Y}^{2}+\mathcal{I}_{X / Y}\right)=\mathcal{I}_{C / Y} / \mathcal{I}_{C / Y}^{2}$. Thus $\mathcal{I}_{C} / \mathcal{I}_{C}^{2}$ is locally free of rank 3. Furthermore, since $\pi$ is a primitive contraction, it must be the blow-up of $X$ along $C$, and so locally $\tilde{X} \subseteq \tilde{Y}$ where $\tilde{Y}$ is the blow-up of $Y$ along $C$. Let $F \subseteq \tilde{Y}$ be the exceptional locus of the blowup of $Y$ along $C ; F$ is isomorphic to the $\mathbf{P}^{2}$-bundle over $C$ given by $\mathbf{P}\left(\mathcal{I}_{C} / \mathcal{I}_{C}^{2}\right)$, and $\mathcal{I}_{F / \tilde{Y}} / \mathcal{I}_{F / \tilde{Y}}^{2}=\mathcal{O}_{F}(1)$. Therefore

$$
\begin{aligned}
\mathcal{I}_{C} / \mathcal{I}_{C}^{2} & =\pi_{*} \mathcal{O}_{F}(1) \\
& =\left.\pi_{*} \mathcal{O}_{F}(1)\right|_{E} \\
& =\pi_{*} \mathcal{I}_{E / \tilde{X}} / \mathcal{I}_{E / \tilde{X}}^{2} \\
& =\pi_{*} \omega_{E}^{-1} .
\end{aligned}
$$

It is easy to see that $R^{1} \pi_{*} \omega_{E}^{-1}=0$, so

$$
\chi\left(\omega_{E}^{-1}\right)=\chi\left(\mathcal{I}_{C} / \mathcal{I}_{C}^{2}\right)=\operatorname{deg}\left(\mathcal{I}_{C} / \mathcal{I}_{C}^{2}\right)+3
$$

as $C \cong \mathbf{P}^{1}$. To compute $\chi\left(\omega_{E}^{-1}\right)$, recall that $E$ has du Val singularities from Theorem 1.1 (b). If $h: \tilde{E} \rightarrow E$ is a minimal resolution, then $h^{*} \omega_{E}^{-1}=\omega_{\tilde{E}}^{-1}$ and so $\chi\left(\omega_{E}^{-1}\right)=\chi\left(\omega_{\tilde{E}}^{-1}\right)-h^{0}\left(R^{1} h_{*} \omega_{\tilde{E}}^{-1}\right)$. Furthermore $R^{1} h_{*} \omega_{\tilde{E}}^{-1}=0$, so $\chi\left(\omega_{E}^{-1}\right)=\chi\left(\omega_{\tilde{E}}^{-1}\right)=K_{\tilde{E}}^{2}+1$, by Riemann-Roch, where $K_{\tilde{E}}$ is the canonical class on $\tilde{E}$. But $K_{\tilde{E}}^{2}=K_{E}^{2}=E^{3}$. We conclude that

$$
\operatorname{deg} \mathcal{I}_{C} / \mathcal{I}_{C}^{2}=E^{3}-2
$$

(c) By $[11$, II 5.10], there is an isomorphism

$$
\begin{aligned}
\mathbf{R} \pi_{*} \underline{\mathbf{R o m}}_{\mathcal{O}_{\tilde{X}}}\left(\pi^{*} \Omega_{X}^{1}, \mathcal{O}_{\tilde{X}}\right) & \cong \mathbf{R} \underline{\operatorname{Hom}}_{\mathcal{O}_{X}}\left(\Omega_{X}^{1}, \mathbf{R} \pi_{*} \mathcal{O}_{\tilde{X}}\right) \\
& \cong \mathbf{R} \underline{\operatorname{Hom}}_{\mathcal{O}_{X}}\left(\Omega_{X}^{1}, \mathcal{O}_{X}\right)
\end{aligned}
$$

The latter isomorphism follows from the fact that $X$ has rational singularities. This yields a spectral sequence

$$
R^{p} \pi_{*} \underline{\operatorname{Ext}}_{\mathcal{O}_{\tilde{X}}}^{q}\left(\pi^{*} \Omega_{X}^{1}, \mathcal{O}_{\tilde{X}}\right) \Rightarrow \underline{\operatorname{Ext}}_{\mathcal{O}_{X}}^{n}\left(\Omega_{X}^{1}, \mathcal{O}_{X}\right)
$$

which gives the exact sequence

$$
0 \rightarrow R^{1} \pi_{*} \underline{\operatorname{Hom}}_{\mathcal{O}_{\tilde{X}}}\left(\pi^{*} \Omega_{X}^{1}, \mathcal{O}_{\tilde{X}}\right) \rightarrow \underline{\operatorname{Ext}}_{\mathcal{O}_{X}}^{1}\left(\Omega_{X}^{1}, \mathcal{O}_{X}\right) \rightarrow \pi_{*} \underline{\operatorname{Ext}}_{\mathcal{O}_{\tilde{X}}}^{1}\left(\pi^{*} \Omega_{X}^{1}, \mathcal{O}_{\tilde{X}}\right) \rightarrow 0 .
$$


This implies (1.4.1). To calculate $\pi_{*} \underline{\operatorname{Ext}}_{\mathcal{O}_{\tilde{X}}}\left(\pi^{*} \Omega_{X}^{1}, \mathcal{O}_{\tilde{X}}\right)$, we use the exact sequence

$$
0 \longrightarrow \mathcal{G} \longrightarrow \pi^{*} \Omega_{X}^{1} \longrightarrow \Omega_{\tilde{X}}^{1} \stackrel{\phi}{\longrightarrow} \Omega_{\tilde{X} / X}^{1} \longrightarrow 0,
$$

where $\mathcal{G}$ is a sheaf supported on $E$. This leads to an exact sequence

$$
0=\underline{\operatorname{Hom}}_{\mathcal{O}_{\tilde{X}}}\left(\mathcal{G}, \mathcal{O}_{\tilde{X}}\right) \rightarrow \underline{\operatorname{Ext}}_{\mathcal{O}_{\tilde{X}}}^{1}\left(\operatorname{ker} \phi, \mathcal{O}_{\tilde{X}}\right) \rightarrow \underline{\operatorname{Ext}}_{\mathcal{O}_{\tilde{X}}}^{1}\left(\pi^{*} \Omega_{X}^{1}, \mathcal{O}_{\tilde{X}}\right)
$$

$$
\rightarrow \underline{\operatorname{Ext}}_{\mathcal{O}_{\tilde{X}}}^{1}\left(\mathcal{G}, \mathcal{O}_{\tilde{X}}\right) \rightarrow \underline{\operatorname{Ext}}_{\mathcal{O}_{\tilde{X}}}^{2}\left(\operatorname{ker} \phi, \mathcal{O}_{\tilde{X}}\right)
$$

and since $\underline{\operatorname{Ext}}_{\mathcal{O}_{\tilde{X}}}^{i}\left(\Omega_{\tilde{X}}^{1}, \mathcal{O}_{\tilde{X}}\right)=0$ for $i>0$, we obtain isomorphisms

$$
\underline{\operatorname{Ext}}_{\mathcal{O}_{\tilde{X}}}^{i}\left(\operatorname{ker} \phi, \mathcal{O}_{\tilde{X}}\right) \cong \underline{\operatorname{Ext}}_{\mathcal{O}_{\tilde{X}}^{i+1}}^{i}\left(\Omega_{\tilde{X} / X}^{1}, \mathcal{O}_{\tilde{X}}\right)
$$

for $i>0$.

To compute $\Omega_{\tilde{X} / X}^{1}$, first note that $\Omega_{\tilde{X} / X}^{1} \otimes_{\mathcal{O}_{\tilde{X}}} \mathcal{O}_{E} \cong \Omega_{E / C}^{1}$. Thus, if

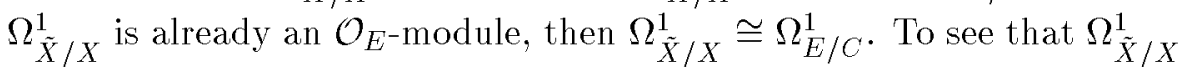
is an $\mathcal{O}_{E}$-module, again locally embed $X$ in a smooth hypersurface $Y$, and let $\tilde{Y} \rightarrow Y$ be the blow-up of $Y$ along $C$, with exceptional divisor $F$. Then $\Omega_{\tilde{Y} / Y}^{1}$ is an $\mathcal{O}_{F}$-module, as can be explicitly calculated, and thus $\Omega_{\tilde{X} / Y}^{1}=\Omega_{\tilde{X} / X}^{1}$ is an $\mathcal{O}_{E-\operatorname{modul}}$.

Now the change of rings spectral sequence ([28, Theorem 11.66]) gives

$$
\underline{\operatorname{Ext}}_{\mathcal{O}_{E}}^{i}\left(\Omega_{E / C}^{1}, \underline{\operatorname{Ext}}_{\mathcal{O}_{\tilde{X}}^{j}}^{j}\left(\mathcal{O}_{E}, \mathcal{O}_{\tilde{X}}\right)\right) \Rightarrow \underline{\operatorname{Ext}}_{\mathcal{O}_{\tilde{X}}}^{n}\left(\Omega_{E / C}^{1}, \mathcal{O}_{\tilde{X}}\right)
$$

and from

$$
0 \rightarrow \mathcal{O}_{\tilde{X}}(-E) \rightarrow \mathcal{O}_{\tilde{X}} \rightarrow \mathcal{O}_{E} \rightarrow 0
$$

we obtain

$$
\underline{\operatorname{Ext}}_{\mathcal{O}_{\tilde{X}}^{j}}^{j}\left(\mathcal{O}_{E}, \mathcal{O}_{\tilde{X}}\right) \cong \begin{cases}\mathcal{O}_{E}(E) \cong \omega_{E} & \text { if } j=1 \\ 0 & \text { if } j \neq 1\end{cases}
$$

so $\operatorname{Ext}_{\mathcal{O}_{\tilde{X}}}^{i+1}\left(\Omega_{\tilde{X} / X}^{1}, \mathcal{O}_{\tilde{X}}\right) \cong \underline{\operatorname{Ext}}_{\mathcal{O}_{E}}^{i}\left(\Omega_{E / C}^{1}, \omega_{E}\right)$. From

$$
0 \rightarrow \pi^{*} \Omega_{C}^{1} \rightarrow \Omega_{E}^{1} \rightarrow \Omega_{E / C}^{1} \rightarrow 0,
$$

it follows that

$$
\begin{aligned}
{\stackrel{\operatorname{Hom}}{\mathcal{O}_{E}}}_{(}\left(\Omega_{E}^{1}, \omega_{E}\right) \stackrel{\pi_{*}}{\longrightarrow} \pi^{*} \mathcal{T}_{C} \otimes \omega_{E} \longrightarrow & \underline{\operatorname{Ext}}_{\mathcal{O}_{E}}^{1}\left(\Omega_{E / C}^{1}, \omega_{E}\right) \\
& \longrightarrow \text { Ext }_{\mathcal{O}_{E}}^{1}\left(\Omega_{E}^{1}, \omega_{E}\right) \longrightarrow 0,
\end{aligned}
$$


and $\operatorname{Ext}_{\mathcal{O}_{E}}^{2}\left(\Omega_{E / C}^{1}, \omega_{E}\right) \cong \operatorname{Ext}_{\mathcal{O}_{E}}^{2}\left(\Omega_{E}^{1}, \omega_{E}\right) \cong 0$ since $E$ has only hypersurface singularities. Thus (1.4.5) yields

$$
0 \rightarrow \underline{\operatorname{Ext}}_{\mathcal{O}_{E}}^{1}\left(\Omega_{E / C}^{1}, \omega_{E}\right) \rightarrow \underline{\operatorname{Ext}}_{\mathcal{O}_{\tilde{X}}}^{1}\left(\pi^{*} \Omega_{X}^{1}, \mathcal{O}_{\tilde{X}}\right) \rightarrow \underline{\operatorname{Ext}}_{\mathcal{O}_{\tilde{X}}}^{1}\left(\mathcal{G}, \mathcal{O}_{\tilde{X}}\right) \rightarrow 0 .
$$

In (1.4.6) $\pi_{*}$ fails to be surjective precisely where $\pi: E \rightarrow C$ is not smooth, and thus coker $\pi_{*}$ has support precisely on this locus. We now use the description of the singularities of $E$ from Theorem $1.1 \mathrm{~b}$ ). It is well-known that at a point $P$ of $E$ which is an $A_{n}$ singularity of $E$,

$$
\operatorname{length}_{P}\left(\underline{\operatorname{Ext}}_{\mathcal{O}_{E}}^{1}\left(\Omega_{E}^{1}, \omega_{E}\right)\right)=n .
$$

Thus, if $\pi: E \rightarrow C$ has a fibre consisting of two $\mathbf{P}^{1}$ 's meeting at a point $P \in E$ with $P$ an $A_{n}$ singularity $(n \geq 0)$ on $E$, then

$$
\text { length }\left(\text { Ext }_{\mathcal{O}_{E}}^{1}\left(\Omega_{E / C}^{1}, \omega_{E}\right)\right) \geq n+1 \text {. }
$$

If, on the other hand, $\pi: E \rightarrow C$ has a fibre which is a double line $l$, then $E$ is locally of the form, in $\mathbf{P}^{2} \times \mathbf{A}^{1}$,

$$
x^{2}+t y z=0
$$

with $\pi$ given by $(x, y, z, t) \mapsto t$. A calculation shows that coker $\pi_{*}$ is an invertible sheaf on the reduced fibre, $\mathbf{P}^{1}$, and thus must be $\mathcal{O}_{\mathbf{P}^{1}} \otimes \omega_{E} \cong$ $\mathcal{O}_{\mathbf{P}^{1}}(-1)$. This gives an exact sequence

$$
0 \rightarrow \mathcal{O}_{\mathbf{P}^{1}}(-1) \rightarrow \underline{\operatorname{Ext}}_{\mathcal{O}_{E}}^{1}\left(\Omega_{E / C}^{1}, \omega_{E}\right) \rightarrow \underline{\operatorname{Ext}}_{\mathcal{O}_{E}}^{1}\left(\Omega_{E}^{1}, \omega_{E}\right) \rightarrow 0 .
$$

Since $E$ has two $A_{1}$ singularities on this fibre, $\pi_{*} \underline{\operatorname{Ext}}_{\mathcal{O}_{E}}^{1}\left(\Omega_{E / C}^{1}, \omega_{E}\right)$ has length 2 at this point. Adding up all contributions, we now see that

$$
\text { length }\left(\pi_{*} \underline{\operatorname{Ext}}_{\mathcal{O}_{E}}^{1}\left(\Omega_{E / C}^{1}, \omega_{E}\right)\right) \geq \operatorname{rank} \operatorname{Pic}(\tilde{E})-2,
$$

where $\tilde{E}$ is a minimal resolution of $E$. This in turn is $8-K_{\tilde{E}}^{2}=8-K_{E}^{2}=$ $8-E^{3}$. Also, $R^{1} \pi_{*}$ Ext $_{\mathcal{O}_{E}}^{1}\left(\Omega_{E / C}^{1}, \omega_{E}\right)=0$. Thus, applying $\pi_{*}$ to $(1.4 .7)$, we obtain

$$
0 \rightarrow \mathcal{F} \rightarrow \pi_{*} \underline{\operatorname{Ext}}_{\mathcal{O}_{\tilde{X}}}^{1}\left(\pi^{*} \Omega_{X}^{1}, \mathcal{O}_{\tilde{X}}\right) \rightarrow \pi_{*} \underline{\operatorname{Ext}}_{\mathcal{O}_{\tilde{X}}}^{1}\left(\mathcal{G}, \mathcal{O}_{\tilde{X}}\right) \rightarrow 0
$$

where $\mathcal{F}=\pi_{*} \operatorname{Ext}_{\mathcal{O}_{E}}^{1}\left(\Omega_{E / C}^{1}, \omega_{E}\right)$ is a sheaf of finite length $\geq 8-E^{3}$. Thus to demonstrate (1.4.2), we only need to calculate $\mathcal{G}$. Again, locally embed $X \subseteq Y, Y$ smooth and dimension 4 . We have an exact sequence

$$
\left.\left.0 \longrightarrow \pi^{*} \mathcal{O}_{Y}(-X)\right|_{X} \stackrel{\psi}{\longrightarrow} \pi^{*} \Omega_{Y}^{1}\right|_{X} \longrightarrow \pi^{*} \Omega_{X}^{1} \longrightarrow 0 .
$$


A local calculation shows that $\psi$ defines a section of $\pi^{*}\left(\left.\Omega_{Y}^{1}\right|_{X} \otimes \mathcal{O}_{Y}(X)\right)$ which vanishes with order one along $E$. Thus the torsion part of coker $\psi$ is an invertible $\mathcal{O}_{E}$-module. Since the torsion part of $\pi^{*} \Omega_{X}^{1}$ is precisely $\mathcal{G}$, this implies that $\mathcal{G}$ is an invertible $\mathcal{O}_{E}$-module.

To determine which invertible $\mathcal{O}_{E}$-module, we tensor (1.4.4) with $\mathcal{O}_{E}$. This yields exact sequences

$$
\begin{array}{r}
\left.\left.\underline{\operatorname{Tor}}_{1}^{\mathcal{O}_{\tilde{X}}}\left(\operatorname{ker} \phi, \mathcal{O}_{E}\right) \rightarrow \mathcal{G} \rightarrow \pi^{*} \Omega_{X}^{1}\right|_{E} \rightarrow(\operatorname{ker} \phi)\right|_{E} \rightarrow 0, \\
\left.\left.0 \rightarrow \underline{\operatorname{Tor}}_{1}^{\mathcal{O}_{\tilde{X}}}\left(\Omega_{E / C}^{1}, \mathcal{O}_{E}\right) \rightarrow(\operatorname{ker} \phi)\right|_{E} \rightarrow \Omega_{\tilde{X}}^{1}\right|_{E} \rightarrow \Omega_{E / C}^{1} \rightarrow 0
\end{array}
$$

and an isomorphism

$$
\underline{\operatorname{Tor}}_{1}^{\mathcal{O} \tilde{X}}\left(\operatorname{ker} \phi, \mathcal{O}_{E}\right) \cong \underline{\operatorname{Tor}}_{2}^{\mathcal{O}} \tilde{x}\left(\Omega_{E / C}^{1}, \mathcal{O}_{E}\right)=0,
$$

the latter equality from

$$
0 \rightarrow \mathcal{O}_{\tilde{X}}(-E) \rightarrow \mathcal{O}_{\tilde{X}} \rightarrow \mathcal{O}_{E} \rightarrow 0
$$

This exact sequence also leads to

$$
\begin{aligned}
0 & \longrightarrow \operatorname{Tor}_{1}^{\mathcal{O}_{\tilde{X}}}\left(\Omega_{E / C}^{1}, \mathcal{O}_{E}\right) \stackrel{\cong}{\longrightarrow} \Omega_{E / C}^{1} \otimes \omega_{E}^{-1} \\
& \longrightarrow \Omega_{E / C}^{1} \stackrel{\cong}{\cong} \Omega_{E / C}^{1} \longrightarrow 0 .
\end{aligned}
$$

We also have an exact sequence

$$
0 \rightarrow \mathcal{I}_{C} /\left.\mathcal{I}_{C}^{2} \rightarrow \Omega_{X}^{1}\right|_{C} \rightarrow \Omega_{C}^{1} \rightarrow 0
$$

injectivity on the left again following from a local calculation showing generic injectivity. Pulling this back to $E$ gives

$$
\left.0 \rightarrow \pi^{*}\left(\mathcal{I}_{C} / \mathcal{I}_{C}^{2}\right) \rightarrow\left(\pi^{*} \Omega_{X}^{1}\right)\right|_{E} \rightarrow \pi^{*} \Omega_{C}^{1} \rightarrow 0 .
$$

Thus

$$
c_{1}\left(\left.\left(\pi^{*} \Omega_{X}^{1}\right)\right|_{E}\right)=\pi^{*} \mathcal{O}_{C}\left(E^{3}-4\right),
$$

from (b). (Here, by $c_{1}$, we really mean $c_{1}$ on the non-singular part of $E$, but we abuse notation and write sheaves on $E$.) From the second sequence of (1.4.8) and (1.4.10),

$$
\begin{aligned}
c_{1}\left(\left.(\operatorname{ker} \phi)\right|_{E}\right) & =c_{1}\left(\Omega_{E / C}^{1} \otimes \omega_{E}^{-1}\right) \otimes c_{1}\left(\left.\Omega_{\tilde{X}}^{1}\right|_{E}\right) \otimes c_{1}\left(\Omega_{E / C}^{1}\right)^{-1} \\
& =\omega_{E}^{-1} .
\end{aligned}
$$


Finally, from the first sequence of (1.4.8) and (1.4.11), we see that $c_{1}(\mathcal{G})=\pi^{*} \mathcal{O}_{C}\left(E^{3}-4\right) \otimes \omega_{E}$. Since $\mathcal{G}$ is invertible,

$$
\mathcal{G}=\pi^{*} \mathcal{O}_{C}\left(E^{3}-4\right) \otimes \omega_{E} .
$$

Thus

$$
\begin{aligned}
\pi_{*} \underline{\operatorname{Ext}}_{\tilde{X}}^{1}\left(\mathcal{G}, \mathcal{O}_{\tilde{X}}\right) & \cong \pi_{*} \underline{\operatorname{Hom}}_{\mathcal{O}_{E}}\left(\mathcal{G}, \omega_{E}\right) \\
& \cong \pi_{*} \pi^{*} \mathcal{O}_{C}\left(4-E^{3}\right) \\
& \cong \mathcal{O}_{C}\left(4-E^{3}\right)
\end{aligned}
$$

which implies (1.4.2).

We have now shown everything in part (c) except that $R^{1} \pi_{*} \underline{\operatorname{Hom}}\left(\pi^{*} \Omega_{X}^{1}, \mathcal{O}_{\tilde{X}}\right)$ is of finite length. Now the singularities of $X$ along $C$ are generically $A_{1}$, and it is well known that at those points of $C$ which are $A_{1}$ singularities, $\mathbf{T}^{1}$ is an invertible $\mathcal{O}_{C}$-module. Hence from (1.4.1) and (1.4.2), $R^{1} \pi_{*} \underline{\operatorname{Hom}}\left(\pi^{*} \Omega_{X}^{1}, \mathcal{O}_{\tilde{X}}\right)$ must be supported on dissident points. q.e.d.

Example 1.5. The above proposition, as well as Theorem 5.2 of [10], leaves open the possibility of a non-normal exceptional divisor $E, E$ rational, with $E^{3}=7$. We give an example of such here. Let $\mathcal{E}=\mathcal{O}_{\mathbf{P}^{1}}(1) \oplus \mathcal{O}_{\mathbf{P}^{1}}(2)^{\oplus 2} \oplus \mathcal{O}_{\mathbf{P}^{1}}$, and let $\mathbf{P}=\mathbf{P}(\mathcal{E})$ be the $\mathbf{P}^{3}$-bundle over $\mathbf{P}^{1}$. If $t=c_{1}\left(\mathcal{O}_{\mathbf{P}}(1)\right)$, then $-K_{\mathbf{P}} \sim 4 t-3 f$. We can write equations on $\mathbf{P}$ using variables $X, Y, Z, W$ corresponding to the inclusions of $\mathcal{O}_{\mathbf{P}^{1}}(1), \mathcal{O}_{\mathbf{P}^{1}}(2), \mathcal{O}_{\mathbf{P}^{1}}(2), \mathcal{O}_{\mathbf{P}^{1}}$ in $\mathcal{E}$ respectively, and variables $u, v$ on $\mathbf{P}^{1}$. Thus

$$
T=\left\{u Y^{2} W^{2}+v Z^{2} W^{2}+X^{3} W=0\right\}
$$

represents an element of $\left|-K_{\mathbf{P}}\right|$, and so $K_{T}=0 . T$ is of course reducible, but is irreducible locally along the line $l=\{X=Y=Z=0\}$. T is also singular along $l$. Blowing up $l$, it is easy to see that this desingularizes $T$ in a neighborhood of $l$, and that the exceptional divisor $E$ is a nonnormal rational surface with $E^{3}=7$. Furthermore, the linear system $|t|$ on $\mathbf{P}$ contracts $l$; this induces a morphism $\pi: T \rightarrow T^{\prime}$, where $\pi(l)$ is an isolated singular point. This provides an example, again locally, of an isolated rational Gorenstein point which is the contraction of a non-normal del Pezzo surface of degree 7.

It is possible to globalize this example by finding a more general element of $\left|-K_{\mathbf{P}}\right|$ with the same local behaviour at $l$, but which is non-singular away from $l$. 
Lemma 1.6. Let $\pi: \tilde{X} \rightarrow X$ be a Type III contraction with normal exceptional locus, $C \cong \mathbf{P}^{1}$ the singular locus of $X$, and suppose $\pi$ is the blow-up of $X$ along $C$. Let $f: \mathcal{X} \rightarrow \Delta$ be a flat deformation of $X$ over a one-dimensional disk $\Delta$. Then either there is a flat deformation $\tilde{f}: \tilde{\mathcal{X}} \rightarrow \Delta$ of $\tilde{X}$, and a map $\pi^{\prime}: \tilde{\mathcal{X}} \rightarrow \mathcal{X}$ over $\Delta$ which is a deformation of $\pi$, or else, after possibly making a finite base-change over $\Delta$, there is a small projective morphism $\mathcal{X}^{\prime} \rightarrow \mathcal{X}$ with $\mathcal{X}^{\prime} \rightarrow \Delta$ flat such that $\mathcal{X}_{t}^{\prime}$ has Q-factorial terminal singularities. In particular, if $\pi$ is primitive, in the latter case $\mathcal{X}^{\prime}=\mathcal{X}$.

Proof. First suppose that each fibre of $f$ has a one-dimensional singular locus. Let $S \subseteq \mathcal{X}$ be the reduced two-dimensional part of the singular locus of $\mathcal{X}$. The central fibre of $S \rightarrow \Delta$ is the curve $C \subseteq X$. If we take a general hyperplane section $\mathcal{H}$ of $\mathcal{X}$, then $\mathcal{H} \rightarrow \Delta$ is a deformation of a hyperplane section $H$ of $X$, which has a certain number of ordinary double points. Since as we deform $H$, the number of ordinary double points cannot go up, we see that $C$ is a reduced fibre of $S \rightarrow \Delta$, and so $S \rightarrow \Delta$ is a non-singular $\mathbf{P}^{1}$-bundle over $\Delta$ near $0 \in \Delta$. Thus if we blow up $S$ inside of $\mathcal{X}$, we obtain $\tilde{f}: \tilde{\mathcal{X}} \rightarrow \Delta$, and the proper transform of $X$ is the blow-up of $X$ along $C$, which is $\tilde{X}$ by hypothesis. Hence $\tilde{f}$ is a deformation of $\tilde{X}$, as desired.

Now suppose that the general fibre of $f$ has codimension- 3 singularities. Since $X$ has cDV singularities, so does the general fibre, and so the general fibre has terminal singularities. Suppose now that the general fibre is not $\mathbf{Q}$-factorial. Let $D_{t}$ be a Weil divisor in a general fibre which is not $\mathbf{Q}$-Cartier. Possibly after making a base change $\Delta^{\prime} \rightarrow \Delta$, we can find a divisor $D$ on $\mathcal{X}$ such that $\left.D\right|_{\mathcal{X}_{t}}=D_{t}$. $D$ is not $\mathbf{Q}$-Cartier. Let

$$
\mathcal{R}_{\mathcal{X}}(D)=\bigoplus_{m=0}^{\infty} \mathcal{O}_{\mathcal{X}}(m D)
$$

If $\mathcal{R}_{\mathcal{X}}(D)$ is a finitely generated $\mathcal{O}_{\mathcal{X}}$-algebra, and we set

$$
\mathcal{X}^{\prime}=\mathbf{P} \operatorname{roj}\left(\mathcal{R}_{\mathcal{X}}(D)\right),
$$

then $\mathcal{X}^{\prime}$ is normal and $\mathcal{X}^{\prime} \rightarrow \mathcal{X}$ is an isomorphism in codimension 1 , by [14, Lemma 3.1]. Furthermore, the proper transform of $D$ is $\mathbf{Q}$-Cartier on $\mathcal{X}^{\prime}$. We can repeat this process until $\mathcal{X}_{t}^{\prime}$ is $\mathbf{Q}$-factorial for general $t \in \Delta$. If $\pi$ is primitive, and $\mathcal{X}_{t}$ were not $\mathbf{Q}$-factorial, then since $\mathcal{X}_{0}^{\prime}$ is a partial resolution of $\mathcal{X}_{0}=X, \mathcal{X}_{0}^{\prime}=\tilde{X}$ or $X$. But $\mathcal{X}_{0}^{\prime} \neq \mathcal{X}_{0}$, so $\mathcal{X}_{0}^{\prime}=\tilde{X}$, which is impossible since then $\mathcal{X}^{\prime} \rightarrow \Delta$ is a flat deformation of $\tilde{X}$ in 
which the exceptional divisor $E \subseteq \tilde{X}$ does not deform. Thus we obtain a contradiction, and so $\mathcal{X}_{0}^{\prime}=X$ and $\mathcal{X}_{t}$ is $\mathbf{Q}$-factorial.

We need then to show that $\mathcal{R}_{\mathcal{X}}(D)$ is finitely generated. We proceed as follows. Working locally, since $\mathcal{X}$ has only cDV singularities, we can write $\mathcal{X}$ as a two-dimensional family of du Val surface singularities $\mathcal{X} \rightarrow S$. By [14, Lemma 3.2], we can replace $\mathcal{X}$ by any finite cover, and thus, in particular, by making a base-change, we can assume that the family $\mathcal{X} \rightarrow S$ has a simultaneous resolution $\mathcal{X}^{\prime} \stackrel{g}{\longrightarrow} \mathcal{X} \rightarrow S$. By taking a general fibration $S \rightarrow S_{1}$, where $S_{1}$ is one-dimensional, we get a fibration $\mathcal{X}^{\prime} \rightarrow S_{1}$ of non-singular threefolds. Since threefold flops exist in families ([17, Theorem 11.10]) we can perform flops on $\mathcal{X}^{\prime}$ until $g^{*} D$ is $g$-nef. $g^{*} D$ then induces a factorization $\mathcal{X}^{\prime} \rightarrow \mathcal{X}^{\prime \prime} \stackrel{h}{\longrightarrow} \mathcal{X}$ with $h^{*} D$ $h$-ample. By [14, Lemma 3.1], it then follows that $\mathcal{R}_{\mathcal{X}}(D)$ is finitely generated. q.e.d.

Theorem 1.7. Let $\pi: \tilde{X} \rightarrow X$ be a primitive type III contraction of a non-singular Calabi-Yau threefold $\tilde{X}$, contracting a divisor $E$ to a curve $C \cong \mathbf{P}^{1}$. Then $X$ is smoothable if $E^{3} \leq 6$.

Proof. Our goal is to find a deformation $\mathcal{X} \rightarrow \Delta$ of $X$ inducing a map $\Delta \rightarrow \operatorname{Def}(X)$ such that the image of $\Delta$ is not contained in $\operatorname{im}(\operatorname{Def}(\tilde{X}) \stackrel{p}{\longrightarrow} \operatorname{De} f(X))$. Here the map $p$, given by blowing-down deformations, exists by $[17,(11.4)]$. If we have such a deformation, then by Lemma 1.6, $\mathcal{X}_{t}$ has $\mathbf{Q}$-factorial terminal singularities for general $t \in \Delta$. It then follows from [20] that $\mathcal{X}_{t}$ is smoothable, so $X$ is smoothable.

Of course, $\operatorname{Def}(\tilde{X})$ is smooth by the Bogomolov-Tian-Todorov unobstructedness theorem. If $\operatorname{De} f(X)$ is smooth, then all we need to do is to show that the differential of $p, p_{*}: T_{\tilde{X}}^{1} \rightarrow T_{X}^{1}$ is a proper inclusion, where $T_{\tilde{X}}^{1}$ and $T_{X}^{1}$ are the tangent spaces of $\operatorname{De} f(\tilde{X})$ and $\operatorname{Def}(X)$ respectively. Since the map $p$ has finite fibres and we can assume $\tilde{X}$ is general in its moduli, we can assume that $p_{*}$ is injective. We need to show that $p_{*}$ is not surjective. As we will see shortly, however, $\operatorname{De} f(X)$ is smooth if $E^{3} \leq 5$, but in the case $E^{3}=6$, this may not be the case.

Step 1. Def $(X)$ is smooth if $E^{3} \leq 5$, and $\operatorname{Def}(X)$ is defined settheoretically by at most one equation in a neighborhood of the origin of $T_{X}^{1}$ if $E^{3}=6$.

Proof. Let $\hat{X}$ be the completion of $X$ along the singular locus $C \subseteq X$. By $\left[10\right.$, Remark 2.7], $\operatorname{Def}(X)$ is smooth if $\operatorname{Ext}_{\mathcal{O}_{X}}^{2}\left(\Omega_{X}^{1}, \mathcal{O}_{\hat{X}}\right)=0$. Note that $\underline{\operatorname{Ext}}_{\mathcal{O}_{X}}^{i}\left(\Omega_{X}^{1}, \mathcal{O}_{\hat{X}}\right)$ is supported on $C$, and $\underline{\operatorname{Ext}}_{\mathcal{O}_{X}}^{2}\left(\Omega_{X}^{1}, \mathcal{O}_{\hat{X}}\right)=0$ since $X$ has only hypersurface singularities. Thus, by the local-global 
spectral sequence for Ext's,

$$
\operatorname{Ext}_{\mathcal{O}_{X}}^{2}\left(\Omega_{X}^{1}, \mathcal{O}_{\hat{X}}\right)=H^{1}\left(\underline{\operatorname{Ext}}_{\mathcal{O}_{X}}^{1}\left(\Omega_{X}^{1}, \mathcal{O}_{\hat{X}}\right)\right) .
$$

Now, locally, if $X \subseteq Y$ with $Y$ four-dimensional and non-singular, we have the sequence

$$
\underline{\operatorname{Hom}}_{\mathcal{O}_{X}}\left(\left.\Omega_{Y}^{1}\right|_{X}, \mathcal{F}\right) \rightarrow \underline{\operatorname{Hom}}_{\mathcal{O}_{X}}\left(\mathcal{I}_{X / Y} / \mathcal{I}_{X / Y}^{2}, \mathcal{F}\right) \rightarrow \underline{\operatorname{Ext}}_{\mathcal{O}_{X}}^{1}\left(\Omega_{X}^{1}, \mathcal{F}\right) \rightarrow 0
$$

for any quasi-coherent sheaf $\mathcal{F}$, or equivalently,

$$
\left.\mathcal{T}_{Y}\right|_{X} \otimes \mathcal{F} \rightarrow \mathcal{N}_{X / Y} \otimes \mathcal{F} \rightarrow \underline{\operatorname{Ext}}_{\mathcal{O}_{X}}^{1}\left(\Omega_{X}^{1}, \mathcal{F}\right) \rightarrow 0 .
$$

This shows that

$$
\underline{\operatorname{Ext}}_{\mathcal{O}_{X}}^{1}\left(\Omega_{X}^{1}, \mathcal{F}\right) \cong \operatorname{Ext}_{\mathcal{O}_{X}}^{1}\left(\Omega_{X}^{1}, \mathcal{O}_{X}\right) \otimes \mathcal{F} .
$$

Thus, since $\underline{\operatorname{Ext}}_{\mathcal{O}_{X}}^{1}\left(\Omega_{X}^{1}, \mathcal{O}_{X}\right)=\mathbf{T}^{1}$ is a coherent sheaf supported on $C$, $\mathbf{T}^{1} \cong \underline{\operatorname{Ext}}_{\mathcal{O}_{X}}^{1}\left(\Omega_{X}^{1}, \mathcal{O}_{\hat{X}}\right)$. By Theorem $1.4(\mathrm{c}), H^{1}\left(\mathbf{T}^{1}\right)=0$ if $E^{3} \leq 5$, and $T_{l o c}^{2}:=\operatorname{Ext}_{\mathcal{O}_{X}}^{2}\left(\Omega_{X}^{1}, \mathcal{O}_{\hat{X}}\right)=H^{1}\left(\mathbf{T}^{1}\right)$ is one-dimensional if $E^{3}=6$. The claim in the case that $E^{3}=6$ then follows from Theorem 2.2 of $[10]$ and Theorem 1 of [15]. q.e.d.

Step 2. $\operatorname{dim} \operatorname{coker}\left(p_{*}: T_{\tilde{X}}^{1} \rightarrow T_{X}^{1}\right) \geq 2$ if $E^{3} \leq 6$.

Proof. By $[31,(1.5)]$, the map $p_{*}$ is the map

$$
\operatorname{Ext}_{\mathcal{O}_{\tilde{X}}}^{1}\left(\Omega_{\tilde{X}}^{1}, \mathcal{O}_{\tilde{X}}\right) \rightarrow \operatorname{Ext}_{\mathcal{O}_{\tilde{X}}}^{1}\left(\pi^{*} \Omega_{X}^{1}, \mathcal{O}_{\tilde{X}}\right)
$$

induced by the natural map $\pi^{*} \Omega_{X}^{1} \rightarrow \Omega_{\tilde{X}}^{1}$; by applying $\mathbf{R} \Gamma$ to both sides of (1.4.3), we see that $\operatorname{Ext}^{1}\left(\pi^{*} \Omega_{X}^{1}, \mathcal{O}_{\tilde{X}}\right) \cong \operatorname{Ext}^{1}\left(\Omega_{X}^{1}, \mathcal{O}_{X}\right)=T_{X}^{1}$.

We set $\mathcal{T}^{\prime}=\underline{\operatorname{Hom}}_{\mathcal{O}_{\tilde{X}}}\left(\pi^{*} \Omega_{X}^{1}, \mathcal{O}_{\tilde{X}}\right)$. The morphism

$$
\mathbf{R} \Gamma \mathbf{R} \underline{\operatorname{Hom}}_{\mathcal{O}_{\tilde{X}}}\left(\Omega_{\tilde{X}}^{1}, \mathcal{O}_{\tilde{X}}\right) \rightarrow \mathbf{R} \Gamma \mathbf{R} \underline{\operatorname{Hom}}_{\mathcal{O}_{\tilde{X}}}\left(\pi^{*} \Omega_{X}^{1}, \mathcal{O}_{\tilde{X}}\right)
$$

induced by $\pi^{*} \Omega_{X}^{1} \rightarrow \Omega_{\tilde{X}}^{1}$ induces a morphism of Grothendieck spectral sequences which gives a diagram: 
Thus dim coker $p_{*} \geq 2$ if

$$
\operatorname{dim} \operatorname{ker} \alpha \leq \operatorname{dim} H^{0}\left(\underline{\operatorname{Ext}}_{\mathcal{O}_{\tilde{X}}}^{1}\left(\pi^{*} \Omega_{X}^{1}, \mathcal{O}_{\tilde{X}}\right)\right)-2
$$

By Theorem $1.4 \mathrm{c}$ ),

$$
\operatorname{dim} H^{0}\left(\underline{\operatorname{Ext}}_{\mathcal{O}_{\tilde{X}}}^{1}\left(\pi^{*} \Omega_{X}^{1}, \mathcal{O}_{\tilde{X}}\right)\right) \geq 8-E^{3}+\operatorname{dim} H^{0}\left(\mathcal{O}_{C}\left(4-E^{3}\right)\right) .
$$

Since we are assuming that $E^{3} \leq 6$, we just need to show that

$$
\operatorname{dim} \operatorname{ker} \alpha \leq \operatorname{dim} H^{0}\left(\mathcal{O}_{C}\left(4-E^{3}\right)\right) .
$$

We will prove this using the sequence (1.4.4). Breaking this up into two short exact sequences, we have first

$$
0 \rightarrow \mathcal{G} \rightarrow \pi^{*} \Omega_{X}^{1} \rightarrow \operatorname{ker} \phi \rightarrow 0 .
$$

Dualising this yields that $\mathcal{T}^{\prime} \cong(\operatorname{ker} \phi)^{\vee}$, while applying $\operatorname{Hom}_{\mathcal{O}_{\tilde{X}}}\left(\cdot, \mathcal{O}_{\tilde{X}}\right)$ gives us a commutative diagram:

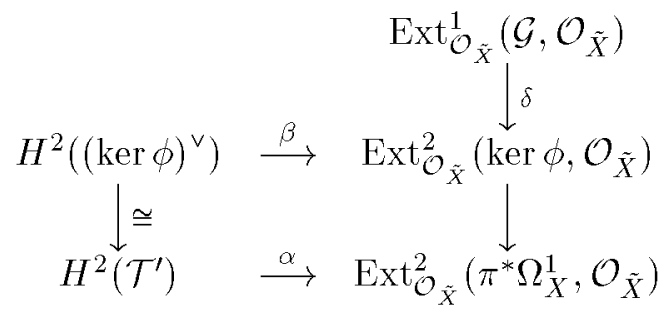


where $\beta$ also comes from the local-global Ext spectral sequence. This shows that if $\beta$ is injective, then $\operatorname{dim} \operatorname{ker} \alpha \leq \operatorname{dim} \operatorname{Ext}_{\mathcal{O}_{\tilde{X}}}^{1}\left(\mathcal{G}, \mathcal{O}_{\tilde{X}}\right)$. But by Serre duality, $\operatorname{Ext}_{\mathcal{O}_{\tilde{X}}}^{1}\left(\mathcal{G}, \mathcal{O}_{\tilde{X}}\right) \cong H^{2}(\mathcal{G})^{\vee}$. From the proof of $\left.1.4 \mathrm{c}\right)$, $\mathcal{G} \cong \pi^{*} O_{C}\left(E^{3}-4\right) \otimes \omega_{E}$, so by Serre duality on $E$,

$$
\begin{aligned}
H^{2}(\mathcal{G})^{\vee} & \cong H^{0}\left(\pi^{*} \mathcal{O}_{C}\left(4-E^{3}\right)\right) \\
& \cong H^{0}\left(\mathcal{O}_{C}\left(4-E^{3}\right)\right) .
\end{aligned}
$$

Hence we see that if $\beta$ is injective, then $\operatorname{dim} \operatorname{ker} \alpha \leq \operatorname{dim} H^{0}\left(\mathcal{O}_{C}\left(4-E^{3}\right)\right)$ as desired.

To show $\beta$ is injective, we use the other piece of the sequence (1.4.4),

$$
0 \rightarrow \operatorname{ker} \phi \rightarrow \Omega_{\tilde{X}}^{1} \rightarrow \Omega_{\tilde{X} / X}^{1} \rightarrow 0 .
$$

Dualising this sequence and using the fact that $\underline{\operatorname{Ext}}_{\mathcal{O}_{\tilde{X}}}^{1}\left(\Omega_{\tilde{X} / X}^{1}, \mathcal{O}_{\tilde{X}}\right) \cong$ $\underline{\operatorname{Hom}}_{\mathcal{O}_{E}}\left(\Omega_{\tilde{X} / X}^{1}, \omega_{E}\right)$, we obtain a commutative diagram with exact rows, setting $\mathcal{T}:=\underline{\operatorname{Hom}}_{\mathcal{O}_{E}}\left(\Omega_{\tilde{X} / X}^{1}, \omega_{E}\right)$,

where the horizontal maps come from the local-global Ext spectral sequence. To show that $\beta$ is injective, it will be enough to show the following claims:

Claim 1. $H^{2}(\mathcal{T})=0$.

Claim 2. $\operatorname{im} \gamma_{1}=\operatorname{im} \gamma_{2}$.

Proof of Claim 1. Since $\mathcal{T}$ restricted to a general fibre $f$ of $\pi: E \rightarrow$ $C$ is $\mathcal{O}_{f}, R^{1} \pi_{*} \mathcal{T}$ is supported on points. Thus by the Leray spectral sequence, $H^{2}(\mathcal{T})=0$. 
Proof of Claim 2. By Serre duality, the first square of (1.7.4) is dual to

$$
\begin{array}{ccc}
\operatorname{Ext}_{\mathcal{O}_{E}}^{1}\left(\left(\Omega_{\tilde{X} / X}^{1}\right)^{\vee}, \mathcal{O}_{E}\right) & \stackrel{\gamma_{1} \vee}{\longleftarrow} & H^{1}\left(\Omega_{\tilde{X}}^{1}\right) \\
\uparrow_{\delta} & & \uparrow= \\
H^{1}\left(\Omega_{\tilde{X} / X}^{1}\right) & \stackrel{\gamma_{2} \vee}{\longleftarrow} & H^{1}\left(\Omega_{\tilde{X}}^{1}\right) .
\end{array}
$$

Clearly $\operatorname{im} \gamma_{1} \subseteq \operatorname{im} \gamma_{2}$. Since $\operatorname{dim} \operatorname{im} \gamma_{i}=\operatorname{dim} \operatorname{im} \gamma_{i}^{v}$, it is enough to show that $\operatorname{dimim} \gamma_{2}{ }^{\vee} \leq \operatorname{dimim} \gamma_{1}{ }^{\vee}$. The local-global Ext spectral sequence gives an inclusion

$$
H^{1}\left(\left(\Omega_{\tilde{X} / X}^{1}\right)^{\vee \vee}\right) \rightarrow \operatorname{Ext}_{\mathcal{O}_{E}}^{1}\left(\left(\Omega_{\tilde{X} / X}^{1}\right)^{\vee}, \mathcal{O}_{E}\right),
$$

through which $\delta$ factors via the natural map

$$
\delta^{\prime}: H^{1}\left(\Omega_{\tilde{X} / X}^{1}\right) \rightarrow H^{1}\left(\left(\Omega_{\tilde{X} / X}^{1}\right)^{\vee \vee}\right) .
$$

Furthermore, $\pi_{*} \Omega_{\tilde{X} / X}^{1}$ and $\pi_{*}\left(\left(\Omega_{\tilde{X} / X}^{1}\right)^{\vee \vee}\right)$ are sheaves of finite length, so

$$
\begin{aligned}
H^{1}\left(\Omega_{\tilde{X} / X}^{1}\right) & =H^{0}\left(R^{1} \pi_{*} \Omega_{\tilde{X} / X}^{1}\right), \\
H^{1}\left(\left(\Omega_{\tilde{X} / X}^{1}\right)^{\vee \vee}\right) & =H^{0}\left(R^{1} \pi_{*}\left(\left(\Omega_{\tilde{X} / X}^{1}\right)^{\vee \vee}\right)\right) .
\end{aligned}
$$

Thus it is enough to show in the diagram

$$
\begin{array}{ccc}
H^{0}\left(R^{1} \pi_{*}\left(\left(\Omega_{\tilde{X} / X}^{1}\right)^{\vee \vee}\right)\right) & \stackrel{\gamma_{1}^{\prime}}{\longleftarrow} & H^{1}\left(\Omega_{\tilde{X}}^{1}\right) \\
\uparrow_{\delta}^{\prime} & & \uparrow= \\
H^{0}\left(R^{1} \pi_{*} \Omega_{\tilde{X} / X}^{1}\right) & \stackrel{\gamma_{2} \vee}{\longleftarrow} & H^{1}\left(\Omega_{\tilde{X}}^{1}\right)
\end{array}
$$

that $\operatorname{dimim} \gamma_{2} \vee \leq \operatorname{dimim} \gamma_{1}^{\prime} . \quad X$ is $\mathbf{Q}$-factorial, as follows from [16, Proposition 5-1-6], because $\pi$ is an extremal divisorial contraction. So by $[10$, Lemma 4.4$]$

$$
\operatorname{im}\left(H^{1}\left(\Omega_{\tilde{X}}^{1}\right) \rightarrow H^{0}\left(R^{1} \pi_{*} \Omega_{\tilde{X}}^{1}\right)\right)=\operatorname{im}\left(\mathbf{C} E \rightarrow H^{0}\left(R^{1} \pi_{*} \Omega_{\tilde{X}}^{1}\right)\right),
$$

and therefore

$$
\operatorname{im}\left(\gamma_{2}^{\vee}\right)=\operatorname{im}\left(\mathbf{C} E \rightarrow H^{0}\left(R^{1} \pi_{*} \Omega_{\tilde{X} / X}^{1}\right)\right) .
$$

This image is one dimensional, and the image of $E$ gives a section of $R^{1} \pi_{*} \Omega_{\tilde{X} / X}^{1}$ which is clearly supported on all of $C$. Since $R^{1} \pi_{*} \Omega_{\tilde{X} / X}^{1}$ and $R^{1} \pi_{*}\left(\left(\Omega_{\tilde{X} / X}^{1}\right)^{\vee \vee}\right)$ differ on only a finite set of points, $\delta^{\prime}(\sigma)$ is non-zero, so $\operatorname{dimim} \gamma_{1}^{\prime} \geq 1$. Hence $1=\operatorname{dim} \operatorname{im}\left(\gamma_{2}^{\vee}\right) \leq \operatorname{dim} \operatorname{im} \gamma_{1}^{\prime}$. 
Step 3. As already observed at the beginning of the proof, Step 2 implies the desired result if $\operatorname{Def}(X)$ is smooth. This is the case if $E^{3} \leq 5$, by Step 1 . If $E^{3}=6$, then $\operatorname{dim} T_{X}^{1} \geq\left(\operatorname{dimim} p_{*}\right)+2$ by Step 2 , and $\operatorname{Def}(X)$ is defined set-theoretically by at most one equation in $T_{X}^{1}$. Thus there still exists a deformation $\mathcal{X} \rightarrow S$ for some $S$ which is at least one dimensional, such that this deformation does not come from a deformation of $\tilde{X}$, and we finish as before. q.e.d.

Remark 1.8. The arguments of Step 1 of the proof of Theorem 1.7 show that $\operatorname{De} f(X)$ can be obstructed if $E^{3}=6,7$ or 8 . However, if $E^{3}=7, T_{l o c}^{2}$ is two-dimensional, yet $H^{0}\left(\mathbf{T}^{1}\right)$ only provides one additional tangent direction. If $E^{3}=8$, in fact $H^{0}\left(\mathbf{T}^{1}\right)=0$. So the $E^{3}=6$ case is the only case where one might expect to see an obstructed deformation space yet still have some actual deformations. Indeed, this is precisely the case for the original example of a Calabi-Yau threefold with canonical singularities and obstructed deformations given in [9]. There, the singular Calabi-Yau threefold has a resolution with exceptional divisor $E$ and $E^{3}=6$.

We can get a stronger result if $E^{3} \leq 4$. The following result should be compared with [10, Theorem 3.8], which implies as a special case that if $\pi: \tilde{X} \rightarrow X$ is a contraction (not necessarily primitive) of a divisor $E$ to a point and $E^{3} \leq 4$, then $X$ is smoothable.

Theorem 1.9. Let $\tilde{X}$ be a non-singular Calabi-Yau threefold, let $\pi: \tilde{X} \rightarrow X$ be a contraction of a normal divisor $E$ to a curve $C \cong \mathbf{P}^{1}$, and assume that $\pi$ is the blow-up of $X$ along $C$. If $E^{3} \leq 4$, then there is a partial resolution $X^{\prime} \rightarrow X$ with $X^{\prime}$ singular, such that $X^{\prime}$ is smoothable.

Proof. We first note that the conclusion about $E$ of Theorem 1.1 b) still holds, since the proof in [32] only uses the fact that $\tilde{X}$ is nonsingular and $\pi$ is the blow-up of $X$ along $C$. The proof of Theorem 1.4 b) and c) only relies on these facts and Theorem $1.1 \mathrm{~b}$ ). So we can still apply these results.

Now suppose we find a deformation $\mathcal{X} \rightarrow \Delta$ of $X$ inducing a map $\Delta \rightarrow \operatorname{De} f(X)$ such that the image of $\Delta$ is not contained in $\operatorname{im}(\operatorname{Def}(\tilde{X}) \stackrel{p}{\longrightarrow} \operatorname{De} f(X))$. Then by Lemma 1.6, we can find, after making a base-change over $\Delta$, a family $\mathcal{X}^{\prime} \rightarrow \Delta$ with $\mathcal{X}^{\prime} \rightarrow \mathcal{X}$ a small morphism and $\mathcal{X}_{t}^{\prime}$ having $\mathbf{Q}$-factorial terminal singularities. Set $X^{\prime}:=\mathcal{X}_{0}^{\prime}$. From [20] it follows that $\mathcal{X}_{t}^{\prime}$, hence $X^{\prime}$, is smoothable. Note that while $X^{\prime} \rightarrow X$ need not itself be a small morphism, $X^{\prime}$ cannot be non-singular; 
otherwise the exceptional divisor would deform in the family $\mathcal{X}^{\prime} \rightarrow \Delta$, and then $\mathcal{X}^{\prime} \rightarrow \mathcal{X}$ would not be a small morphism.

Now, since $E^{3} \leq 4$, Step 1 of the proof of Theorem 1.7 implies that $\operatorname{De} f(X)$ is smooth and so we only need to show that $p_{*}: T_{\tilde{X}}^{1} \rightarrow T_{X}^{1}$ is not a surjection. As in Step 2 of the proof of Theorem 1.7, we need to prove that

$$
\operatorname{dim} \operatorname{ker} \alpha<\operatorname{dim} H^{0}\left(\underline{\operatorname{Ext}}_{\mathcal{O}_{\tilde{X}}}^{1}\left(\pi^{*} \Omega_{X}^{1}, \mathcal{O}_{\tilde{X}}\right)\right) .
$$

By Theorem $1.4 \mathrm{c}$ ),

$$
\operatorname{dim} H^{0}\left(\underline{\operatorname{Ext}}_{\mathcal{O}_{\tilde{X}}}^{1}\left(\pi^{*} \Omega_{X}^{1}, \mathcal{O}_{\tilde{X}}\right)\right)=\operatorname{length}(\mathcal{F})+\operatorname{dim} H^{0}\left(\mathcal{O}_{C}\left(4-E^{3}\right)\right) .
$$

Since $E^{3} \leq 4, \operatorname{dim} H^{0}\left(\mathcal{O}_{C}\left(4-E^{3}\right)\right)>0$, so we just need to show

$$
\operatorname{dim} \operatorname{ker} \alpha \leq \text { length }(\mathcal{F}) .
$$

Suppose in diagram (1.7.2), the map $\delta$ was zero. Then $\operatorname{dim} \operatorname{ker} \alpha=$ $\operatorname{dim} \operatorname{ker} \beta$, and it would be enough to show that $\operatorname{dim} \operatorname{ker} \beta \leq$ length $(\mathcal{F})$. But by the proof of Theorem 1.4 (c), $\underline{\operatorname{Ext}}_{\mathcal{O}_{\tilde{X}}}^{2}\left(\operatorname{ker} \phi, \mathcal{O}_{\tilde{X}}\right)=0$, and $H^{1}\left(\underline{\operatorname{Ext}}_{\mathcal{O}_{\tilde{X}}}^{1}\left(\operatorname{ker} \phi, \mathcal{O}_{\tilde{X}}\right)\right)=0$, so the local-global Ext spectral sequence yields an exact sequence

$$
H^{0}\left(\underline{\operatorname{Ext}}_{\mathcal{O}_{\tilde{X}}}^{1}\left(\operatorname{ker} \phi, \mathcal{O}_{\tilde{X}}\right)\right) \rightarrow H^{2}\left((\operatorname{ker} \phi)^{\vee}\right) \stackrel{\beta}{\longrightarrow} \operatorname{Ext}_{\mathcal{O}_{\tilde{X}}}^{2}\left(\operatorname{ker} \phi, \mathcal{O}_{\tilde{X}}\right) \rightarrow 0
$$

Again from the proof of Theorem $1.4 \mathrm{c}$ ),

$$
\operatorname{dim} H^{0}\left(\underline{\operatorname{Ext}}_{\mathcal{O}_{\tilde{X}}}^{1}\left(\operatorname{ker} \phi, \mathcal{O}_{\tilde{X}}\right)\right)=\operatorname{length}(\mathcal{F}),
$$

so we see $\operatorname{dim} \operatorname{ker} \beta \leq$ length $(\mathcal{F})$. Thus we just need to show

Claim. The map $\delta$ in $(1.7 .2)$ is zero.

Proof. The Serre dual of $\delta$ is the boundary map

$$
H^{1}(\operatorname{ker} \phi) \stackrel{\delta^{\vee}}{\longrightarrow} H^{2}(\mathcal{G})
$$

induced by (1.7.1), so it is enough to show that $\delta^{\vee}$ is zero, or equivalently, that

$$
H^{1}\left(\pi^{*} \Omega_{X}^{1}\right) \stackrel{\xi}{\longrightarrow} H^{1}(\operatorname{ker} \phi)
$$

is surjective. Now since $H^{0}\left(\Omega_{\tilde{X} / X}^{1}\right)=H^{0}\left(\Omega_{E / C}^{1}\right)=0,(1.7 .3)$ gives the exact sequence

$$
0 \rightarrow H^{1}(\operatorname{ker} \phi) \rightarrow H^{1}\left(\Omega_{\tilde{X}}^{1}\right) \rightarrow H^{1}\left(\Omega_{E / C}^{1}\right)
$$


If we identify $H^{1}\left(\Omega_{\tilde{X}}^{1}\right)$ with $\operatorname{Pic} \tilde{X} \otimes \mathbf{C}$, this implies that $H^{1}(\operatorname{ker} \phi)$ is generated by divisors which are all $\pi$-numerically trivial; i.e., divisors which are pullbacks of divisors on $X$. Thus we have a commutative diagram

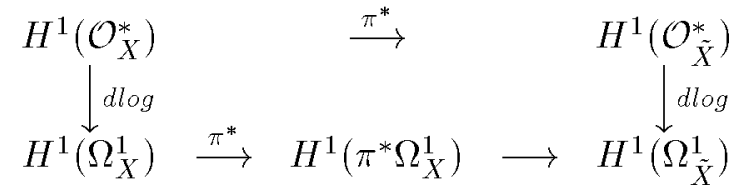

and the image of the composed map $H^{1}\left(\mathcal{O}_{X}^{*}\right) \rightarrow H^{1}\left(\Omega_{\tilde{X}}^{1}\right)$ generates $H^{1}(\operatorname{ker} \phi)$. Hence $\xi$ is surjective. q.e.d.

\section{The Kähler cone of primitive Calabi-Yau threefolds}

Having now understood which primitive contractions yield smoothable Calabi-Yau threefolds, we would like to get a feeling of what kind of constraints this information imposes on primitive Calabi-Yaus. As a first example, recall the following theorem of Nikulin:

Theorem 2.1. Suppose $X$ is a non-singular Calabi-Yau threefold. Then one of the following holds:

(1) $\operatorname{rank} \operatorname{Pic}(X) \geq 41$.

(2) X has a small contraction.

(3) There exists a nef $\mathbf{R}$-divisor $D \in \operatorname{Pic}(X) \otimes \mathbf{R}$ with $D^{3}=0$.

The number 41 is probably far from being optimal. However, the constraints imposed on primitive Calabi-Yaus easily lead to the following optimal result.

Theorem 2.2. Suppose $X$ is a non-singular primitive Calabi-Yau threefold. Then one of the following holds:

(1) $\operatorname{rank} \operatorname{Pic}(X)=1$.

(2) X has a small contraction.

(3) There exists a nef $\mathbf{R}$-divisor $D \in \operatorname{Pic}(X) \otimes \mathbf{R}$ with $D^{3}=0$.

Proof. Suppose that neither case (2) or (3) occur. By [32], the closure of the Kähler cone $\overline{\mathcal{K}}$ of $X$ is rational polyhedral, and every 
codimension-one face induces a primitive divisorial contraction. Following the notation of [21], let $R_{1}, \ldots, R_{m}$ be the extremal rays of $N E(X)$, the cone of effective curves, and denote by $D\left(R_{i}\right)$ the divisor contracted by contracting the extremal ray $R_{i}$. By [21, Theorem $\left.1.3 .2(1)\right]$, the $D\left(R_{i}\right)$ are all distinct divisors. Now let $\left\{R_{i}\right\}_{i \in I}$ be an $E$-set. (See [21, $\S 1.1]$ for the definition of an E-set.) Then by Theorem 1.2.13 of [21], $\left\{R_{i}\right\}_{i \in I}$ satisfies condition (iii) of $\S 1.1$ of [21], so there is a non-zero nef divisor $D=\sum_{i \in I} a_{i} D\left(R_{i}\right)$ with $a_{i} \geq 0$. Now according to Theorem 0.4 and Theorem 5.8 of $[10], D\left(R_{i}\right)$ is rational and satisfies $D\left(R_{i}\right)^{3} \geq 7$; otherwise the contraction induced by $R_{i}$ would yield a smoothable singular Calabi-Yau threefold. It then follows as in the proof of [10, Theorem $5.2]$ that $c_{2}(X) \cdot D\left(R_{i}\right)=12-2 D\left(R_{i}\right)^{3}<0$, so that $c_{2}(X) . D<0$, but this is impossible, since $c_{2}$ is non-negative on the Kähler cone of $X$.

q.e.d.

Let us speculate about each case in turn.

(1) Every Picard number 1 Calabi-Yau is obviously primitive. Some completely new idea will be needed to understand such threefolds. I hope that these threefolds will prove to form a more manageable class.

(2) There are examples of primitive Calabi-Yau threefolds with small contractions.

Example 2.3. Take a general anti-canonical hypersurface in the $\mathbf{P}^{3}$ bundle $\mathbf{P}\left(\mathcal{O}_{\mathbf{P}^{1}}(-1) \oplus \mathcal{O}_{\mathbf{P}^{1}}^{\oplus 2} \oplus \mathcal{O}_{\mathbf{P}^{1}}(1)\right.$ ) over $\mathbf{P}^{1}$. (See [9], [29] for details on this threefold.) Such a Calabi-Yau has Picard number 2, and the two boundaries of the Kähler cone correspond to a K3 fibration and a small contraction of a single $\mathbf{P}^{1}$ to an ordinary double point. However, if we flop this $\mathbf{P}^{1}$, we obtain a non-primitive Calabi-Yau which has a contraction of a rational ruled surface $E$ to a $\mathbf{P}^{1}$ with $E^{3}=-2$.

So we might ask for yet a stronger condition:

Definition 2.4. A Calabi-Yau threefold $X$ is birationally primitive if every minimal model of $X$ is primitive.

I do not know the answer to

Question 2.5. Can a birationally primitive Calabi-Yau threefold have more than one minimal model?

I would hesitate to turn this into a conjecture at this point; it could merely reflect an ignorance of examples.

(3) is the case we know the most about. It appears to be quite a reasonable conjecture that if there exists a nef $\mathbf{R}$-divisor $D$ with $D^{3}=0$, 
then there is a nef $\mathbf{Q}$-divisor with the same property. This would follow from some cone conjectures: see [35] for a survey of these conjectures. If $D$ is a nef divisor with $D^{3}=0$, and $D \cdot c_{2}>0$, then $|n D|$ induces a $\mathrm{K} 3$ or elliptic fibration for sufficiently large $n$ (see [22]). If $D \cdot c_{2}=0$, then we do not know yet whether $|n D|$ will always induce a fibration (elliptic or abelian), but see [34] for results in this direction. In general one should expect to obtain such a fibration.

Once we know a Calabi-Yau has a fibration, this gives us a great deal of information. For example, up to birational equivalence, there are only a finite number of elliptic Calabi-Yau threefolds [8]. Given the classification methods known for elliptic fibrations, I believe it would be a quite tractable task to find all primitive elliptic Calabi-Yau fibrations. Perhaps K3 and abelian surface fibrations will prove to be similarly tractable.

Example 2.6. It is often harder to tell if a Calabi-Yau is primitive than to show one is not primitive. Some Calabi-Yau threefolds are obviously primitive, as mentioned in the introduction: if a Calabi-Yau has no birational contractions, then it is primitive. This includes examples such as hypersurfaces of bidegree $(3,3)$ in $\mathbf{P}^{2} \times \mathbf{P}^{2}$, double covers of $\mathbf{P}^{1} \times \mathbf{P}^{2}$ and double covers of $\mathbf{P}^{1} \times \mathbf{P}^{1} \times \mathbf{P}^{1}$, as well as any Picard number 1 threefold. Example 2.3 yields a primitive Calabi-Yau which does have a birational contraction. However, if the Picard number is much larger than 2, then there could be many birational contractions, most of them not primitive contractions, and at the moment we cannot really check each of these models for smoothability. However, we can give some likely candidates for primitive Calabi-Yaus with larger Picard number.

Let $S$ be one of the rational scrolls $F_{12}, F_{8}, F_{6}$ or $F_{4}$. Let $X_{12}, X_{8}, X_{6}$ and $X_{4}$ be elliptic fibrations over each of these surfaces respectively given by Weierstrass equation

$$
Y^{2}=X^{3}+a X+b
$$

with general $a \in \Gamma\left(S, \omega_{S}^{\otimes-4}\right), b \in \Gamma\left(S, \omega_{S}^{\otimes-6}\right)$. It is easy to check that these are Calabi-Yau threefolds, and have non-singular minimal models. Let $C_{0}$ be the negative section of $S$. Then over $C_{0}$, the fibration $X_{12} \rightarrow S$ has fibres of Kodaira type $I I^{*}$, the fibration $X_{8} \rightarrow S$ has fibres of Kodaira type $I I I^{*}$, the fibration $X_{6} \rightarrow S$ has fibres of Kodaira type $I V^{*}$, and the fibration $X_{4} \rightarrow S$ has fibres of Kodaira type $I_{0}^{*}$, and in each case the inverse image of $C_{0}$ is a union of minimal rational scrolls. We 
have $\rho\left(X_{12}\right)=11, \rho\left(X_{8}\right)=10, \rho\left(X_{6}\right)=9$ and $\rho\left(X_{4}\right)=7$. Furthermore, each of these Calabi-Yaus has only one minimal model. Every primitive contraction is a type III contraction of a minimal rational scroll, which will be either one of the scrolls in the inverse image of $C_{0}$ or the unique section of the fibration. It seems very likely that each of these CalabiYaus is primitive. $X_{12}$ is of special interest since $\chi\left(X_{12}\right)=-960$, and this is apparently the smallest known Euler characteristic of a CalabiYau threefold. If $X_{12}$ were not primitive, we would expect to find a Calabi-Yau with even smaller Euler characteristic.

\section{Relating primitive Calabi-Yau threefolds to general Calabi-Yau threefolds}

Our goal is to shed some light on Questions 0.3, and their relationship to the classification of primitive Calabi-Yaus. In particular, we would like to explore possible corollaries to the conjecture that there are only a finite number of families of primitive or birationally primitive Calabi-Yaus. We first need to consider the question of deformation invariance of the Kähler cone for Calabi-Yau threefolds with canonical singularities. In particular, let $\mathcal{X} \rightarrow \Delta$ be a one-parameter smoothing of a Calabi-Yau $X$ with canonical singularities. We would like to compare the Kähler cone of $\mathcal{X}_{0}, \overline{\mathcal{K}}_{0}$, with the Kähler cone of $\mathcal{X}_{t}, \overline{\mathcal{K}}_{t}$, for general $t$. Now, in general, the Picard numbers $\rho\left(\mathcal{X}_{0}\right)$ might be smaller than $\rho\left(\mathcal{X}_{t}\right)$. For example, if $X=\mathcal{X}_{0}$ is obtained by contracting an elliptic scroll on $\tilde{X}$ to a curve, then $\rho\left(\mathcal{X}_{t}\right)=\rho(\tilde{X})$, while $\rho(X)=\rho(\tilde{X})-1$. The problem here is that we contracted "too much". We can deform $\tilde{X}$ to the same threefolds which we can deform $X$ to. So there was no need to contract the elliptic scroll. More generally, if we wish to make a birational contraction $\pi: \tilde{X} \rightarrow X$ and then smooth $X$, then we should choose $\pi$ to be a "minimal" such contraction which yields the desired smoothing. We make this precise below.

Proposition 3.1. Let $f: \mathcal{X} \rightarrow \Delta$ be a deformation of a Calabi-Yau $X$ with canonical singularities, and suppose that $f: \mathcal{X} \rightarrow \Delta$ satisfies the condition

(3.1.1) For $p: S \rightarrow \Delta$ any morphism, $\mathcal{X}_{S}=\mathcal{X} \times{ }_{\Delta} S$, and for any Weil divisor $D$ on $\mathcal{X}_{S}$ which is Cartier outside finitely many fibres, $D$ is $\mathbf{Q}$-Cartier.

Then, shrinking $\Delta$ if necessary, the Picard number of $\mathcal{X}_{t}$ is constant for $t \in \Delta$, and $\left(R^{1} f_{*} \mathcal{O}_{\mathcal{X}}^{*}\right) \otimes_{\mathbf{Z}} \mathbf{Q} \cong R^{2} f_{*} \mathbf{Q}$ is a constant sheaf on $\Delta$. 
Proof. If $f: \mathcal{X} \rightarrow \Delta$ satisfies the hypotheses, then it satisfies conditions (12.2.1.1)-(12.2.1.3) of [17]. The result thus follows from $[17,12.2 .5]$ and the fact that $H^{1}\left(\mathcal{O}_{\mathcal{X}_{t}}\right)=H^{2}\left(\mathcal{O}_{\mathcal{X}_{t}}\right)=0$ for all $t \in \Delta$. We have to tensor with $\mathbf{Q}$ as the specialization of a Cartier divisor might only be $\mathbf{Q}$-Cartier. q.e.d.

Given an arbitrary smoothing $f: \mathcal{X} \rightarrow \Delta$, can we modify it so that it satisfies condition (3.1.1)? For this, we need

Definition. Let $X$ be a normal variety. A $\mathbf{Q}$-factorialization $\pi: \bar{X} \rightarrow X$ is an isomorphism in codimension one with $\bar{X}$ normal such that $\bar{X}$ is $\mathbf{Q}$-factorial.

Proposition 3.2. Let $f: \mathcal{X} \rightarrow \Delta$ be a one-parameter smoothing of a Calabi-Yau $X$. Then there is a finite morphism $\Delta^{\prime} \rightarrow \Delta$ such that if $\overline{\mathcal{X}} \rightarrow \mathcal{X}_{\Delta^{\prime}}$ is a $\mathbf{Q}$-factorialization then $\overline{\mathcal{X}} \rightarrow \Delta^{\prime}$ satisfies condition (3.1.1).

Proof. Shrinking $\Delta$ if necessary, $\left.R^{1} f_{*} \mathcal{O}_{\mathcal{X}}^{*}\right|_{\Delta-0}$ is a local system which by $[17,12.2 .5]$ has finite monodromy. Thus, we can make a finite base-change $\Delta^{\prime} \rightarrow \Delta$ ramified only at 0 such that $\left.R^{1} f_{*} \mathcal{O}_{\mathcal{X}_{\Delta^{\prime}}^{*}}\right|_{\Delta^{\prime}-0}$ is a constant local system. It is hence clear that if $\overline{\mathcal{X}} \rightarrow \mathcal{X}_{\Delta^{\prime}}$ is a $\mathbf{Q}-$ factorialization of $\mathcal{X}_{\Delta^{\prime}}$, then $\overline{\mathcal{X}} \rightarrow \Delta$ satisfies (3.1.1). q.e.d.

If $f: \mathcal{X} \rightarrow \Delta$ is a family satisfying (3.1.1), then we can compare the Kähler cones in the family. If $f$ is a smoothing of $X=\mathcal{X}_{0}$, then by [32] we can assume that $\overline{\mathcal{K}}_{t}$ is constant for $t \neq 0$, by shrinking $\Delta$ if necessary. We thus have $\overline{\mathcal{K}}_{0} \subseteq \overline{\mathcal{K}}_{t}$. Now we know from [32] and [19] that equality can fail to hold if $\mathcal{X}_{0}$ contains certain types of ruled surfaces. However, when $\mathcal{X}_{0}$ has canonical singularities, the situation is worse: even small contractions in $\mathcal{X}_{0}$ may not deform to the general fibre.

Example 3.3. Let $P_{1}=\mathbf{P}\left(\mathcal{O}_{\mathbf{P}^{1}}^{\oplus 4}\right)=\mathbf{P}^{1} \times \mathbf{P}^{3}$ and let

$$
P_{2}=\mathbf{P}\left(\mathcal{O}_{\mathbf{P}^{1}}(-1) \oplus \mathcal{O}_{\mathbf{P}^{1}}^{\oplus 2} \oplus \mathcal{O}_{\mathbf{P}^{1}}(1)\right) \text {. }
$$

Let $C \subseteq P_{2}$ be the section of $P_{2}$ corresponding to the surjection

$$
\mathcal{O}_{\mathbf{P}^{1}}(-1) \oplus \mathcal{O}_{\mathbf{P}^{1}}^{\oplus 2} \oplus \mathcal{O}_{\mathbf{P}^{1}}(1) \rightarrow \mathcal{O}_{\mathbf{P}^{1}}(-1)
$$

By [9], it is possibly to construct a family $\mathcal{X} \rightarrow \Delta$ where $\mathcal{X}_{0}$ is an anticanonical hypersurface in $P_{2}$ with double points along $C$, and $\mathcal{X}_{t}$ for $t \neq 0$ is a non-singular anticanonical hypersurface in $P_{1}$. If we write Pic $\mathcal{X}_{t}=\mathbf{Z} T \oplus \mathbf{Z} F$, where $T$ is the restriction of $c_{1}\left(\mathcal{O}_{P_{i}}(1)\right)$ to $\mathcal{X}_{t}$ and 
$F$ the restriction of a fibre of $P_{i} \rightarrow \mathbf{P}^{1}$, then the Kähler cone of $\mathcal{X}_{0}$ is spanned by $F$ and $T+F$, the latter divisor contracting $C$, while the Kähler cone of $\mathcal{X}_{t}, t \neq 0$ is spanned by $T$ and $F$. This shows that the Kähler cone might fail to be constant because a small contraction fails to deform.

To rectify this problem, we need the existence of fourfold flops. Recall

Definition. Let $X^{-}$be a normal variety and $D$ a $\mathbf{Q}$-Cartier divisor on $X^{-}$, and let $\phi: X^{-} \rightarrow Y$ be a birational contraction which is an isomorphism in codimension one with $-D \phi$-ample and $K_{X}$ - $\phi$-trivial. The $D$-flop of $\phi$ is a normal variety $X^{+}$and a diagram

with $\psi$ a birational map, $\phi^{+}$an isomorphism in codimension one and $\psi(D) \phi^{+}$-ample.

We note that existence of flops for fourfolds is not known. However, it is known ([18]) that there is no infinite sequence of $D$-flops on a fourfold with terminal singularities.

If fourfold flops exist, however, then we can proceed as follows. If $D$ is a divisor on $\mathcal{X}$, with $D_{t} \in \overline{\mathcal{K}}_{t}, t \neq 0$, then there is a family $\mathcal{X}^{\prime} \rightarrow \Delta$ related by flops over $\Delta$ to $\mathcal{X}$ with $\mathcal{X}_{0}^{\prime}$ birationally equivalent to $\mathcal{X}_{0}$, such that $D$ is nef. We use this for

Theorem 3.4. Suppose Q-factorializations and flops exist for fourfolds with terminal singularities. Then every Calabi-Yau threefold $\tilde{X}$ with Q-factorial terminal singularities has a minimal model which is a crepant $\mathbf{Q}$-factorial terminal resolution of a Calabi-Yau $X$, with $X$ the central fibre of a family $\mathcal{X} \rightarrow \Delta$ and $\mathcal{X}_{t}$ a non-singular birationally primitive Calabi-Yau for $t \neq 0$.

Proof. We proceed by induction on $\rho(\tilde{X})$. If $\tilde{X}$ is birationally primitive, we are done. This is in particular the case if $\rho(\tilde{X})=1$.

Suppose $\tilde{X}$ is a non-singular, non-birationally primitive Calabi-Yau. Then, changing the minimal model of $\tilde{X}$ if necessary, there is a contraction $\tilde{X} \rightarrow X$ and a smoothing $\mathcal{X} \rightarrow \Delta$ of $X$. By Proposition 3.2, after 
making a base-change, we can assume $\mathcal{X} \rightarrow \Delta$ satisfies (3.1.1), and we can then replace $\mathcal{X}$ with its $\mathbf{Q}$-factorialization. This might change $\mathcal{X}_{0}$, but it will still be birational to $\tilde{X}$. We replace $X$ by $\mathcal{X}_{0}$. By Proposition 3.1, $\rho\left(\mathcal{X}_{t}\right)=\rho\left(\mathcal{X}_{0}\right)=\rho(X)<\rho(\tilde{X})$ for general $t \in \Delta$. If $\mathcal{X}_{t}$ is birationally primitive, we are again done. If not, by induction there is a minimal model $\mathcal{X}_{t}^{\prime}$ of $\mathcal{X}_{t}$, a family $\mathcal{Y} \rightarrow \Delta^{\prime}$ with $\mathcal{X}_{t}^{\prime} \rightarrow \mathcal{Y}_{0}$ a crepant resolution, with $\mathcal{Y}_{t}$ birationally primitive. Let $H$ be an ample divisor on $\mathcal{Y}$, which we pull back to $H \in \operatorname{Pic} \mathcal{X}_{t}^{\prime}$, where $H$ is nef. Identifying $\operatorname{Pic} \mathcal{X}_{t}$ with $\operatorname{Pic} \mathcal{X}_{t}^{\prime}$, we denote also by $H \in \operatorname{Pic} \mathcal{X}_{t}$ the proper transform of $H$ on $\mathcal{X}_{t} . H$ is in the moving cone of $\mathcal{X}_{t}$ (see [14] for the definition of the moving cone). By Proposition 3.1, $H$ defines a Q-Cartier Weil divisor on $\mathcal{X}$. Since $\mathcal{X}_{0}$ has canonical singularities and $\mathcal{X}_{t}$ is smooth, $\mathcal{X}$ has terminal singularities by [30]. By performing fourfold flops on $\mathcal{X}$ over $\Delta$, we can find a family $\mathcal{X}^{\prime} \rightarrow \Delta$ birationally equivalent to $\mathcal{X} \rightarrow \Delta$ such that $H$ is nef on $\mathcal{X}^{\prime}$. $H$ then induces a contraction $\mathcal{X}^{\prime} \rightarrow \mathcal{X}^{\prime \prime}$ over $\Delta$ such that $\mathcal{X}_{t}^{\prime \prime} \cong \mathcal{Y}_{0}$. Since $\mathcal{Y}_{0}$ can be deformed to a birationally primitive Calabi-Yau, $\mathcal{X}_{0}^{\prime \prime}$ can also be deformed to a birationally primtive Calabi-Yau. This gives the desired family.

If $\tilde{X}$ is singular with $\mathbf{Q}$-factorial terminal singularities, then by [20], we can smooth $\tilde{X}$, obtaining a family $\mathcal{X} \rightarrow \Delta$ whose general fibre is smooth and $\mathcal{X}_{0}=\tilde{X}$. We then proceed as before. q.e.d.

This answers Question (1) of the introduction, assuming the existence of a finite classification of (birationally) primitive Calabi-Yaus and an understanding of fourfold birational geometry. What are the obstacles to answering the other questions given this information?

First, in passing from Question (1) to Question (2), let us try to apply the same proof as in Theorem 3.4. We immediately run into trouble, since when we pass to the $\mathbf{Q}$-factorialization, $\tilde{X}$ may no longer be a resolution of $\mathcal{X}_{0}$. Secondly, when we perform flops on $\mathcal{X}$, this might also change the birational model of $\mathcal{X}_{0}$, and $\mathcal{X}_{0}$ may no longer have $\tilde{X}$ as a resolution. Thus some better understanding of the birational geometry involved is necessary.

We now discuss Question (3). Fix a non-singular Calabi-Yau threefold $X$. We would like to know that $X$ has a finite number of degenerations. In other words, we would like a scheme $\mathcal{S}$ of finite type, and a flat family $\mathcal{X} \rightarrow \mathcal{S}$ of Calabi-Yau threefolds with canonical singularities such that for any family $\mathcal{X}^{\prime} \rightarrow \Delta$ with $\mathcal{X}_{t}^{\prime}$ deformation equivalent to $X, \mathcal{X}_{0}^{\prime}$ is birational to $\mathcal{X}_{s}$ for some $s \in \mathcal{S}$. To construct $\mathcal{S}$, choose an ample divisor $H$ on $X$. Suppose that there is an integer $r$ satisfying the 
condition

(3.5) For every family $f: \mathcal{X}^{\prime} \rightarrow \Delta$ with $\mathcal{X}_{t}^{\prime}$ deformation equivalent to $X$ and $\mathcal{X}_{0}^{\prime}$ having canonical singularities and such that the divisor $H$ on $\mathcal{X}_{t}^{\prime}$ extends to a $\mathbf{Q}$-Cartier $f$-ample divisor $\mathcal{H}$ on $\mathcal{X}^{\prime}, r \mathcal{H}$ is Cartier.

If this is the case, then we can just take $\mathcal{S}$ to be a suitable open subset of the irreducible component of the Hilbert scheme containing a point corresponding to $X$ embedded via $10 r H$, and $\mathcal{X} \rightarrow \mathcal{S}$ the universal family. Thus given any family $f: \mathcal{X}^{\prime} \rightarrow \Delta$, with $\mathcal{X}_{t}^{\prime}$ deformation equivalent to $X$, we can, after making a base-change, a $\mathbf{Q}$-factorialization and some flops, assume that $\mathcal{X}^{\prime}$ has an $f$-ample $\mathbf{Q}$-Cartier divisor $\mathcal{H}$ extending $H$. So $r \mathcal{H}$ is Cartier, and by [23], $10 r \mathcal{H}$ is $f$-very ample, and hence $\mathcal{X}_{0}^{\prime}=\mathcal{X}_{s}$ for some $s \in \mathcal{S}$.

Unfortunately, it is not clear when (3.5) holds. $\mathcal{X}$ has fourfold terminal Gorenstein singularities. [24] gives a sequence of fourfold terminal Gorenstein singularities with Q-Cartier Weil divisors $D$ such that the minimum $r$ such that $r D$ is Cartier is unbounded. So it may be possible that for larger and larger values of $r$ in (3.5), one will get more and more possible degenerations. Thus, in particular, even if there are only a finite number of families of primitive Calabi-Yau threefolds, it still may be possible that they might have an infinite number of degenerations, causing problems for Questions (3) and (4).

One possible way around this problem could come from a greater understanding of the path one follows from an arbitrary Calabi-Yau to a primitive Calabi-Yau. For example, in $\S 2$, we obtained the combinatorial restrictions of Theorem 2.2 only by analysing smoothability of primitive contractions. Suppose every non-primitive Calabi-Yau threefold $\tilde{X}$ has a primitive contraction $\pi: \tilde{X} \rightarrow X$ with $X$ smoothed by a family $\mathcal{X} \rightarrow \Delta$. It is easy to understand the index of a $\mathbf{Q}$-Cartier divisor on $\mathcal{X}$ since $\mathcal{X}$ will have relatively simple terminal singularities, and so we would be able to get around the problem of (3.5) by limiting attention to such simpler degenerations. But at this point this is mere speculation. A deeper analysis of the Kähler cone of primitive Calabi-Yau threefolds or a deeper analysis of degenerations of Calabi-Yau threefolds is needed.

\section{References}

[1] A. Avram, P. Candelas, D. Jancić \& M. Mandelberg, On the connectedness of the moduli space of Calabi-Yau manifolds, Nuclear Phys. B 465 (1996) 458-472. 
[2] P. Candelas, P. Green \& T. Hübsch, Rolling among Calabi-Yau vacua, Nuclear Phys. B 330 (1990) 49-102.

[3] T. Chiang, B. Greene, M. Gross \& Y. Kanter, Black hole condensation and the Web of Calabi-Yau manifolds, Nuclear Phys. B (Proc. Suppl.) 46 (1996) 82-95.

[4] R. Friedman, Simultaneous resolution of threefold double points, Math. Ann. 274 (1986) 671-689.

[5] P. Green \& T. Hübsch, Possible phase transitions among Calabi-Yau compactifications, Phys. Rev. Lett. 61 (1988) 1163-1166.

[6] Connecting moduli spaces of Calabi-Yau threefolds, Comm. Math. Phys. 119 (1988) 431-441.

[7] B. Greene, D. Morrison, \& A. Strominger, Black hole condensation and the unification of string vacua, Nuclear Phys. B 451 (1995) 109-120.

[8] M. Gross, A finiteness theorem for elliptic Calabi-Yau threefolds, Duke. Math. J. 74 (1994) 271-299.

[9] The deformation space of Calabi-Yau n-folds with canonical singularities can be obstructed, to appear in Essays in Mirror Symmetry II.

[10] Deforming Calabi-Yau threefolds, to appear in Math. Ann.

[11] R. Hartshorne, Residues and duality, Lecture Notes in Math. Vol 20, Springer, Berlin, 1966.

[12] Algebraic geometry, Springer, Berlin,1977.

[13] Y. Kawamata, Minimal models and the Kodaira dimension of algebraic fiber spaces, J. Reine Angew. Math. 363 (1985) 1-46.

[14] The Crepant blowing-up of 3-dimensional canonical singularities and its application to the degeneration of surfaces, Ann. of Math. 127 (1988) 93-163.

[15] U Unobstructed deformations II, J. Algebraic Geom. 3 (1995) 277-280.

[16] Y. Kawamata, K. Matsuda, \& K. Matsuki, Introduction to the minimal model program, Algebraic Geometry, Sendai, Adv. Stud. Pure Math., Vol. 10, (T. Oda, ed.) Kinokuniya-North-Holland, 1987, 283-360.

[17] J. Kollár \& S. Mori, Classification of three-dimensional fips, J. Amer. Math. Soc. 5 (1992) 533-703.

[18] K. Matsuki, Termination of flops for 4-folds, Amer. J. Math. 113 (1991) 835-859.

[19] Y. Namikawa, On deformations of Calabi-Yau 3-folds with terminal singularities, Topology 33 (1994) 429-446.

[20] Y. Namikawa \& J. Steenbrink, Global smoothing of Calabi-Yau three-folds, to appear in Invent. Math. 
[21] V. Nikulin, Diagram method for 3-folds and its application to Kähler Cone and Picard number of Calabi-Yau 3-folds I, preprint, 1993.

[22] K. Oguiso, On algebraic fiber space structures on a Calabi-Yau 3-fold, Internat. J. Math. 4 (1993) 439-465.

[23] K. Oguiso, \& T. Peternell, On polarized canonical Calabi-Yau threefolds, Math. Ann. 301 (1995) 237-248.

[24] S. Onn \& B. Sturmfels, A note on lattice simplices and toric varieties, Amer. J. Math. 116 (1994) 1337-1339.

[25] Z. Ran, Deformations of Maps, Algebraic Curves and Projective Geometry, (E. Ballico, C. Ciliberto, eds.) Lecture Notes in Math. Vol. 1389, Springer, Berlin, 1989.

[26] M. Reid, Canonical 3-folds, Géométrie Algébrique Angers, (A. Beauville, ed.), Sijthoff+Noordhoof, Alphrann der Rijn, 1980, 273-310.

[27] The moduli space of 3-folds with $K=0$ may nevertheless be irreducible, Math. Ann. 278 (1987) 329-334.

[28] J. Rotman, An introduction to homological algebra, Academic Press, New York, 1979.

[29] Y. Ruan, Topological sigma model and Donaldson type invariants in Gromov theory, preprint, 1993.

[30] J. Stevens, On canonical singularities as total spaces of deformations, Abh. Math. Sem. Univ. Hamburg 58 (1988) 275-283.

[31] J. Wahl, Equisingular deformations of normal surface singularities, I, Ann. of Math. 104 (1976) 325-356.

[32] P. M. H. Wilson, The Kähler Cone on Calabi-Yau threefolds, Invent. Math. 107 (1992) 561-583.

[33] Erratum to The Kähler Cone on Calabi-Yau threefolds, Invent. Math. 114 (1993) 231-233.

[34] - The existence of elliptic fibre space structures on Calabi-Yau threefolds, Math. Ann. 300 (1994) 693-703.

[35] - The role of $c_{2}$ in Calabi-Yau classification: a preliminary survey, to appear in Essays on Mirror Symmetry II, Internat. Press, Boston.

[36] Symplectic deformations of Calabi-Yau threefolds, preprint, 1995. 